\title{
Simplifying products of fractional powers of powers
}

\author{
David R. Stoutemyer*
}

March 8, 2012

\begin{abstract}
Most computer algebra systems incorrectly simplify

$$
\frac{z-z}{\frac{\sqrt{w^{2}}}{w^{3}}-\frac{1}{w \sqrt{w^{2}}}}
$$
\end{abstract}

to 0 rather than to $0 / 0$. The reasons for this are:

1. The default simplification doesn't succeed in simplifying the denominator to 0 .

2. There is a rule that 0 is the result of 0 divided by anything that doesn't simplify to either 0 or $0 / 0$.

Many of these systems have more powerful optional transformation and general purpose simplification functions. However that is unlikely to help this example even if one of those functions can simplify the denominator to 0 , because the input to those functions is the result of default simplification, which has already incorrectly simplified the overall ratio to 0 . Try it on your computer algebra systems!

This article describes how to simplify products of the form $w^{\alpha}\left(w^{\beta_{1}}\right)^{\gamma_{1}} \cdots\left(w^{\beta_{n}}\right)^{\gamma_{n}}$ correctly and well, where $w$ is any real or complex expression and the exponents are rational numbers.

It might seem that correct good simplification of such a restrictive expression class must already be published and/or built into at least one widely used computeralgebra system, but apparently this issue has been overlooked. Default and relevant optional simplification was tested with 86 examples on 5 systems with $n=1$. Using a spectrum from the most serious flaw being a result that is not equivalent to the input somewhere to the least serious being not rationalizing a denominator when that doesn't cause a more serious flaw, the overall percentage of most flaw types is alarming:

\begin{tabular}{|c||c|c|c|c|c|c|c|c|}
\hline flaw: & $\not \equiv$ & 0-recognition & $\begin{array}{c}\text { cancelable } \\
\text { singularity }\end{array}$ & $\begin{array}{c}\text { extra } \\
\text { factor }\end{array}$ & excessive $\left|\gamma_{k}\right|$ & $\neg$ canonical & $\neg$ idempotent & $\frac{\ldots}{\sqrt{\ldots}}$ \\
\hline \hline$\%:$ & 11 & 50 & 25 & 16 & 32 & 39 & 0.4 & 6 \\
\hline
\end{tabular}

*dstout at hawaii dot edu 


\section{Introduction}

"When you are right you cannot be too radical;"

- Martin Luther King Jr.

First, a few crucial definitions:

Definition. Default simplification is what a computer-algebra system does to a standard mathematical expression when the user presses ENTER or SHIFT ENTER, using factory-default mode settings without enclosing the expression in an optional transformational function such as expand(...), factor(...), or simplify(...).

Default simplification is the minimal set of transformations that a system does routinely. Default simplification is called evaluation in Mathematica ${ }^{\circledR}$ and in some other systems. Any fixed set of default transformations is likely to omit ones that are wanted in some situations and to include ones that are unwanted in other situations. Therefore:

- Most systems also provide optional transformations done by a function such as expand (...) or by assigning a certain value to a control variable such as trigExpand $\leftarrow$ true.

- Some systems provide a way to disable default transformations. For example the Maxima assignment simp : false suppresses most simplification, whereas the Maxima box(...), Mathematica Hold[...] and Maple freeze(...) functions suppress most or all transformations on their argument.

Definition. Simplification is idempotent for a class of input expressions if simplification of the result (by the same default or optional transformations) yields the same result.

Definition. A conveniently cancelable singularity is a removable singularity that can be removed exactly by functional identities such as $\sin (2 w) \equiv 2 \sin (w) \cos (w)$ together with transformations such as a common denominator followed by factoring out the gcd of any resulting numerator and denominator, then using the law of exponents $w^{\mu} w^{\nu} \rightarrow w^{\mu+\nu}$.

For example, $z^{3} z^{-2} \rightarrow z, \sin (2 z) / \sin (z) \rightarrow 2 \cos (z)$, and

$$
\frac{1}{c(c x-1)}+\frac{1}{c} \rightarrow \frac{x}{c x-1},
$$

which cancels the removable singularity at $c=0$, leaving the non-removable singularity along the hyperbola $c x=1$. However the removable singularity in $\sin (z) / z$ is not conveniently cancelable because it can't be canceled exactly except inconveniently by means such as introducing the piecewise function

$$
\frac{\sin (z)}{z} \rightarrow \begin{cases}1, & \text { if } z=0 \\ \frac{\sin (z)}{z}, & \text { otherwise }\end{cases}
$$

or the infinite series

$$
\frac{\sin (z)}{z} \rightarrow \sum_{k=0}^{\infty} \frac{(-1)^{k} z^{2 k}}{(2 k+1) !}
$$


Definition. A nested power product is an expression or a sub-expression of the form

$$
w^{\alpha}\left(w^{\beta_{1}}\right)^{\gamma_{1}} \cdots\left(w^{\beta_{n}}\right)^{\gamma_{n}},
$$

with $n \geq 1$, rational exponents, and $\alpha$ possibly 0 or 1 .

This article describes simple algorithms that can be used in default and/or optional transformations to simplify nested power products correctly and well. The abstract presents one example of why this is important.

Default and relevant optional transformations for Derive ${ }^{\circledR}$ 6.00, TI-CAS version 3.101, Maxima 5.24.0, Maple ${ }^{\mathrm{tm}} 15.00$ and Mathematica 8.0.4.0 were tested on 86 examples for the simplest case where $n=1$. The table in the Abstract shows the overall percentages for each of eight different decreasingly serious flaw types described in Section 3 .

Those large percentages for the six most serious kinds of flaws are alarming, and so are many corresponding percentages for each of the five systems 2 Wikipedia currently lists 29 other computer algebra systems, and I strongly suspect that most or all of them also have substantial room for improvement in this regard.

Here is an outline of the rest of the article: Section 2 defines three more crucial terms. Section 3 describes eight prioritized goals for results that are nested power products, why they are important, and the reasons for their priorities. Section 4 describes the tables of results at the end of this article and how the listed result flaws were measured. Section 5 describes four good forms for nested power products and how to obtain them:

1. Form 1 merely standardizes the outer fractional exponents to the interval $(-1,1)$ in a way that doesn't introduce removable singularities, but instead tends to reduce their magnitude - perhaps completely.

2. Form 2 further reduces many outer fractional exponents to $[-1 / 2,1 / 2]$ in a way that cancels as much of any removable singularity as can be done without resorting to form 4 . Form 2 is an improvement on form 1 at the expense of more computation.

3. Form 3 absorbs $w^{\alpha}$ into one of the nested powers just prior to display if $w^{\alpha}$ can thus be totally absorbed, giving a result with one less factor. Form 3 is an aesthetic improvement on form 2 at the expense of more computation.

4. Form 4 completely cancels any cancelable singularity and nicely collapses all of the exponents into a single unnested exponent. However, this form often entails a complicated unit magnitude piecewise constant factor that is -1 raised to a complicated exponent. Unsophisticated users might be baffled by this factor, and even sophisticated users might abhor the mess. However, this form must be addressed because it can occur in input, it is valuable for some purposes, and some computer algebra systems generate this form for some inputs.

\footnotetext{
${ }^{1}$ The computer algebra embedded in a succession of TI handheld calculators, Windows and Macintosh computers has no name independent of the product names, the most recent of which is TI-Nspire ${ }^{\text {tm }}$.

${ }^{2}$ I am guilty as a coauthor of Derive and TI-computer algebra.
} 
Section 6 suggests how to extend the algorithms to recognize syntactically different but equivalent instances of $w$ in nested power products and how to extend the algorithms to some kinds of non-numeric exponents. Section 7 is an overall summary. The Appendix lists about one page of Mathematica rewrite rules that implement most of the third result form. Tables of results and their flaw numbers for the five systems and for the rewrite rules are at the end of the article.

\section{More key definitions}

In this article:

- Unless stated otherwise, an indeterminate is a variable that has no assigned value, rather than a result such as $0 / 0$.

- Any finite or infinite-magnitude complex value can be substituted for indeterminates in expressions.

- Fractional powers and square roots denote the principal branch 3

Definition. A canonical form for a class of expressions is one for which all equivalent expressions in the class are represented uniquely.

Canonical forms help make cancellations of equivalent sub-expressions automatic. For example, if a computer algebra system always makes arguments of functional forms such as $\sin (\ldots)$ canonical, then an input sub-expression such as $\sin \left((x+1)^{2}\right)-\sin \left(x^{2}+2 x+1\right)$ automatically simplifies to 0 rather than remaining unchanged as a bulky land mine that might make a subsequent result incorrect. Without canonical arguments, recognition of cryptically similar factors and terms requires costly tests such as determining if the difference in corresponding arguments can be simplified to 0. This might happen every time the same two functional forms meet during processes such as expansion of an integer power of a sum containing two sines, which can be often. In contrast, canonical arguments permit a much faster mere syntactic comparison of functional forms.

As discussed in [1, 6, 10], canonical forms are unnecessarily costly and rigid for the entire class of expressions addressed by general-purpose computer algebra systems. However, canonical forms are acceptable and good for default simplification of some simple classes of irrational sub-expressions such as nested power products.

Definition. Zero-recognizing simplification for a class of expressions is simplification for which all expressions in the class equivalent to 0 are transformed to 0.

As illustrated by the example in the Abstract, a failure to recognize that a subexpression is equivalent to 0 can lead to dramatically incorrect results. Therefore it is desirable for default simplification to have at least a zero-recognition property. It has

\footnotetext{
${ }^{3}$ By default some systems assume that indeterminates represent real values and/or use the real branch wherein for reduced integers $m$ and $n,(-1)^{m / n} \rightarrow 1$ for $m$ even, and $(-1)^{m / n} \rightarrow-1$ for $m$ and $n$ odd. However, most computer algebra systems provide a way to force the principal branch if it isn't the default - and to declare that an indeterminate is complex if that isn't the default.
} 
been proven impossible to guarantee this even for some rather simple classes of irrational expressions, but a strong effort should be made to achieve at least zero recognition for as broad a class of expressions as is practical.

Definition. Candid simplification produces results that are not equivalent to an expression that visibly manifests a simpler expression class.

For example, in a candid result there are no superfluous variables, degree magnitudes are not larger than necessary, there are no unnecessary irrational sub-expressions, and irrationalities are nested no more deeply than necessary. Thus without being as rigidly constrained as canonical forms, candid simplification yields more desirable properties than mere zero-recognizing simplification.

Definition. In this article undefined means an unknown point in the entire infinite complex plane, such as the result of $0 / 04$

Definition. A conveniently representable subset of the infinite complex plane is one that is reasonably representable using constant expressions extended by sets, intervals and the symbol $\infty$.

Conveniently representable proper subsets of the infinite complex plane are regarded here as defined. Particular computer algebra systems might not be able to represent the full range of possibilities, but this article is suggesting what should be done as well as reporting the current situation. These ideas are discussed in more detail in [9], but for this article the major defined subset of interest that isn't a single point is the result of $u / 0$ for any particular non-zero complex constant $u$. This result should be some representation of complex infinity. Among many other benefits it permits the correct computation

$$
\frac{1}{1+\frac{1}{0}} \rightarrow 0 .
$$

Does your computer algebra system do this?

- For Mathematica, 1/0 $\rightarrow$ ComplexInfinity.

- For Derive, with its default real domain, $1 / 0 \rightarrow \pm \infty$.

- For TI-CAS, regrettably $1 / 0 \rightarrow$ undef.

\footnotetext{
${ }^{4}$ It is of course audacious to define undefined. Although unnecessary for this article, systems could usefully also

- display $0 / 0$ as $0 / 0$ rather than a vague controversial word such as "undefined", and

- contract functions of $0 / 0$ to strict subsets of the complex plane wherever possible, such as $\arg (0 / 0) \rightarrow(-\pi, \pi]$. Having $\arg (0 / 0) \rightarrow 0 / 0$ snatches defeat from the jaws of compromise. Try this on your systems! Many systems throw an error, which is worse because it requires even amateur authors of functions to know about all the potential throws, catch them or vet to prevent them, and respond appropriately to make their functions robust.
} 
- Maxima and Maple inconveniently throw an error.

When a proper subset of the infinite complex plane isn't conveniently representable, then the next best thing is to degrade it to $0 / 0$. However, that shouldn't be done for subsets that are as easily represented as complex infinity.

If finite or infinite magnitude complex numbers are substituted for all of the indeterminates in an unsimplified input expression, then that input expression is undefined at that point if and only if the result is $0 / 0$.

Definition. A generalized limit is the set of uni-directional limits of an input expression from all possible directions in the complex plane.

When the generalized limit of an input expression at a conveniently cancelable singularity is a conveniently representable proper subset of the entire infinite complex plane, then this article regards it as not only acceptable but commendable to cancel the singularity and thereby produce a result expression whose substitutional value is that conveniently representable subset at that point.

Reasons for this attitude about mathematics software include:

- Otherwise the results tend to be unacceptably complicated 5

- There is a high likelihood that the physical problem is actually continuous there too - Nature abhors a removable singularity. Removable singularities are often an artifact of the modeling such as using a polar or spherical coordinate system.

- Cancelable singularities are often a result of an unnecessary previous transformation unavoidably done by a system (such as inappropriate rationalization of a denominator) or a result of a previous transformation such as monic normalization, a tangent half angle substitution, or expansion into partial fractions deemed necessary to obtain an anti-derivative.

- Cancellation to simplify nested power products is consistent with quiet transformations such as $w / w \rightarrow 1$ that are currently unavoidable in most computer algebra systems;

- Symbolic cancelation tends to reduce rounding errors near removable singularities for subsequent substitution of floating-point numbers.

However, this transformation of expressions has the composability consequence that substitution of numeric values doesn't necessarily commute with simplification. To accommodate either treatment of expressions, computer algebra systems could and should build in provisos such as " $\mid w \neq 0$ " that are optionally attached automatically to intermediate and final results containing canceled removable singularities, as suggested in [3, 10]. Meanwhile, implementers who do not want to completely cancel cancelable singularities for simplifying nested power products can adapt the algorithms presented here to merely reduce the magnitude of cancelable singularities, such as $\left(z^{2}\right)^{5 / 2} / z^{3} \rightarrow\left(z^{2}\right)^{3 / 2} / z$ rather than transforming all the way to $z \sqrt{z^{2}}$.

\footnotetext{
${ }^{5}$ Canceling a gcd occasionally increases bulk significantly, such as $\left(x^{99}-1\right) /(x-1) \rightarrow x^{98}+x^{97}+\cdots+$ $x+1$, but the algorithms described here consider only syntactic cancellation, which always decreases bulk.
} 


\section{A list of goals for simplifying nested power products}

The most important concern is correctness, followed by candidness, then aesthetics and compliance with custom. More specifically, here is a list of desirable but partially conflicting goals for simplifying nested power product and their differences, in decreasing order of importance:

1. The result should be equivalent to the input wherever the input is defined. (It is acceptable for the result to be a generalized limit of the input where the input is $0 / 0$.)

2. A linear combination of two or more equivalent nested power products should simplify to a multiple of a single power product - or to 0 if the linear combination is equivalent to 0 .

3. Let the net exponent of $\left(w^{\beta_{k}}\right)^{\gamma_{k}}$ be

$$
\triangle_{k}:=\beta_{k} \gamma_{k}
$$

and for a product of nested powers of $w$ let the total positive nested exponent and the total negative nested exponent be

$$
\begin{aligned}
& \triangle_{+}:=\sum_{k=1}^{n} \max \left(\triangle_{k}, 0\right) \\
& \triangle_{-}:=\sum_{k=1}^{n} \min \left(\triangle_{k}, 0\right)
\end{aligned}
$$

When possible, use the transformation

$$
\left(w^{\beta_{k}}\right)^{\gamma_{k}} \rightarrow w^{m_{k} \beta_{k}}\left(w^{\beta_{k}}\right)^{\gamma_{k}-m_{k}}
$$

with appropriate integers $m_{k}$ to minimize $\min \left(\max (\alpha, 0)+\triangle_{+},-\min (\alpha, 0)-\triangle_{-}\right)$, thus canceling as much of any removable singularity as is possible by this means.

4. When possible, fully absorb the $w^{\alpha}$ into the nested powers of $\left(w^{\beta_{k}}\right)^{\gamma_{k}}$ to have fewer factors.

5. Otherwise use transformation (44) to minimize $\triangle_{+}-\triangle_{-}$to minimize the contributions of the troublesome nested powers.

6. Inputs that are equivalent where both are defined should produce the same (canonical) result.

7. Results should be idempotent: Reapplying the same default or optional simplification to the result should leave it unchanged.

8. To help achieve goal 6 , rationalize a denominator in a nested power product when this doesn't introduce a removable singularity or increase its magnitude. 
A larger numbered goal should not be fulfilled if the only way to fulfill it is to violate a smaller numbered goal. For example, fulfillment of goals 5 or 8 can often violate goals 1 , 2 and/or 3.

The reasons for this ranking of the goals are:

1. A violation of goal 1 is most unsatisfactory because it is a result that is not equivalent to the input everywhere the input is defined. For example, if expression $w$ can be 0 , then rationalizing the denominator of $1 / \sqrt{w}$ to give $\sqrt{w} / w$ makes an input that is a well-defined complex infinity at $w=0$ become $0 / 0$ there. A more serious example is the mal-transformation $\left(w^{-2}\right)^{-1 / 2} \rightarrow\left(w^{2}\right)^{1 / 2}$, because the two sides differ along the entire positive and negative imaginary axis. For example $\left(i^{-2}\right)^{-1 / 2}=-i$, whereas $\left(i^{2}\right)^{1 / 2}=i$.

2. The example in the Abstract shows the importance of zero recognition. For example if default simplification of one nested power product produces $\sqrt{z} / z$ and default simplification of another nested power product produces the equivalent expression $z / \sqrt{z}$, then the latter violates goal 8 and together they violate goal 6 . These violations are minor; but if default simplification doesn't simplify their difference to 0 , then that is a violation of goal 2 , which is serious.

3. A violation of goal 3 is next most serious because it is a squandered opportunity to improve the result by canceling a conveniently cancelable singularity and thereby making the result have the limiting value at $w=0$ rather than be undefined there. For example,

$$
\begin{aligned}
\frac{\left(w^{2}\right)^{5 / 2}}{w} & \rightarrow \frac{w^{2}\left(w^{2}\right)^{3 / 2}}{w} \rightarrow w\left(w^{2}\right)^{3 / 2} \\
w\left(\frac{1}{w^{2}}\right)^{5 / 2} & \rightarrow \frac{w}{w^{2}}\left(\frac{1}{w^{2}}\right)^{3 / 2} \rightarrow \frac{1}{w}\left(\frac{1}{w^{2}}\right)^{3 / 2} .
\end{aligned}
$$

4. A violation of goal 4 is more complicated than need be. For example, most people would agree that $w^{4}\left(w^{2}\right)^{1 / 2}$ is more complicated than $\left(w^{2}\right)^{5 / 2}$, which has one less factor.

5. Goal 5 is important because when there is more than one factor of the form $\left(w^{\beta}\right)^{\gamma_{k}}$, there might be more than one way to distribute only some of $w^{\alpha}$ into the nested powers. In contrast if $\gamma_{k}$ is a half-integer power then there are only two ways to minimize $\left|\gamma_{k}\right|$ by factoring an integer power of $w^{\alpha}$ out of $\left(w^{\alpha}\right)^{\gamma}$, or only one way for other fractional powers. Moreover, unnested exponents are less specialized and can therefore interact more freely with other factors in a product. For example, for intermediate results (5) and (6),

$$
\begin{aligned}
w\left(w^{2}\right)^{3 / 2} & \rightarrow w^{2}\left(w^{2}\right)^{1 / 2} \\
\frac{1}{w}\left(\frac{1}{w^{2}}\right)^{3 / 2} & \rightarrow \frac{1}{w^{2}}\left(\frac{1}{w^{2}}\right)^{1 / 2} .
\end{aligned}
$$


6. For a given $w$, the above goals tend to yield the most concise possible nested power product in terms of $w$. Therefore it is better to have the consistency of having two inputs that are equivalent where they are defined return the same most concise form. More importantly a canonical form for nested power product sub-expressions greatly facilitates achieving goal 2 - a major benefit for very little effort.

7. Without idempotency, an unaware user could obtain inconsistent results, and a cautious aware user would have to re-enter such results as inputs until they cycle or stop changing.

8. Goal 8 complies with the custom of rationalizing denominators and helps achieve canonicality goal 6 . For example,

$$
\frac{w}{\sqrt{w^{2}}} \rightarrow \frac{w \sqrt{w^{2}}}{w^{2}} \rightarrow \frac{\sqrt{w^{2}}}{w} .
$$

But rationalization should not be done at the expense of lower-numbered goals. For example,

$$
\frac{1}{w\left(w^{2}\right)^{2 / 3}} \nrightarrow \nrightarrow \frac{\left(w^{2}\right)^{1 / 3}}{w^{3}},
$$

because although it reduces the absolute value of the outer exponent (goal 5), it violates goal 1 by making an input that is complex infinity at $w=0$ become a result that is $0 / 0$ there.

\section{Important information about the tables}

Tables at the end of this article show the results that occurred for each example with each system and with the Appendix rewrite rules. In all of the tables the goal numbers in the Section 3 goals that aren't satisfied but could be satisfied without violating a lowernumbered goal are listed beside each result. Unmet goal numbers 1 and 2 are boldface to emphasize their extreme seriousness.

\subsection{Examples, test protocol and table interpretation}

Tables 2 through 7 report default and relevant optional transformation results for test family 1: multiplying $w^{m}$ by $\left(w^{2}\right)^{n+2 / 3}$ for successive integer $m=-3$ through 3 in combination with successive integer $n=-3$ through 2. For comparison with results that meet all of the goals, Table 1 has corresponding results for form 3 described in Section 5, as produced by the one page of Mathematica rewrite rules listed in the Appendix.

Tables 9 through 15 report default and relevant optional transformation results for test family 2: multiplying $w^{m}$ by $\left(w^{-2}\right)^{n+1 / 2}$ for the same combinations of $m$ and $n$. For comparison with results that meet all of the goals, Table 8 has corresponding form 3 results produced by the Appendix rewrite rules. 
To help assess compliance with goal 8, Table 16 compares results for all of the systems with the Appendix rewrite rules on the particularly simple input $w / \sqrt{w^{2}}$. Some of the examples in test families 1 and 2 also test this goal.

Table 17 tests only whether or not the expression

$$
\frac{\sqrt{w^{2}}}{w}-(-1)^{\frac{1}{2}\left(\arg \left(w^{2}\right)-2 \arg (w)\right) / \pi}
$$

simplifies to 0 . This is the difference between equivalent expressions in form 3 and form 4. This is a more difficult but not impossible problem. All systems fail - including the Appendix rewrite rules, which do not address this issue.

Here is how compliance with the goals was assessed:

1. Most of the results that violated goal 1 did so only at $w=0$. However, the goal 1 violations in Tables 13 and 14 instead or also are not equivalent to the input where it is defined along the entire positive and negative imaginary axis. This is caused by an outlaw of exponents: transforming $\left(w^{-\lambda}\right)^{\mu}$ to $\left(w^{\lambda}\right)^{-\mu}$ for fractional $\mu$, which is not valid along these semi-axes.

2. For test family 1, each input is equivalent to the input two rows down and one column left wherever both are defined, and their omnidirectional limits are identical wherever one of the inputs is $0 / 0$. Therefore to assess compliance with goal 2 (zero-recognition), for every entry in the table I computed the difference $w^{m}\left(w^{2}\right)^{n+2 / 3}-w^{m+2}\left(w^{2}\right)^{n-1+2 / 3}$ or the optional transformation thereof and the difference $w^{m}\left(w^{2}\right)^{n+2 / 3}-w^{m-2}\left(w^{2}\right)^{n+1+2 / 3}$, then considered it a flaw for the entry if either of these two differences was non-zero. Thus compliance with this goal is not discernible from merely inspecting the result entries. Compliance is a property of the default simplification or optional transformation when given the difference of two non-identical but equivalent nested power products. For test family 2, each input is equivalent to the input two rows down and one column right wherever both are defined, so I did an analogous test for that. It is of course possible for an entry to pass these limited tests but fail for more widely separated equivalent inputs 6 For Table 16 the result is equivalent to $\sqrt{w} / w$ and $w^{2} /\left(w^{2}\right)^{3 / 2}$, so I tested whether or not the corresponding two differences or optional transformation thereof simplified to 0 . Table 17 tests zero recognition directly, and only than - but with only one particular difference in equivalent forms rather than only two.

3. To comply with goal 3 without violating lower-numbered goals, a result $w^{\hat{\alpha}}\left(w^{\beta}\right)^{\hat{\gamma}}$

\footnotetext{
${ }^{6}$ This happens for Tables 13 and [14] All of the columns would exhibit flaw 2 if one of the two equivalent expressions was always taken from the correct results in columns 3 or 4 . The results are not equivalent to the inputs for columns 1,2,5 and 6 , so the only reason the difference simplified to 0 for columns 1 and 6 was the subtraction of incorrect but identical results - an instance where two wrongs make a right.
} 
should have

$$
\begin{gathered}
\alpha=0 \vee \\
(\operatorname{sign}(\hat{\alpha})=\operatorname{sign}(\beta \hat{\gamma}) \wedge|\hat{\gamma}|<1) \vee \\
(\alpha \neq 0 \wedge \operatorname{sign}(\hat{\alpha}) \neq \operatorname{sign}(\beta \hat{\gamma}) \wedge|\hat{\gamma}|<1 \wedge \\
\min (|\beta \hat{\gamma}|,|\hat{\alpha}|) \leq \min (|\beta(\hat{\gamma}-\operatorname{sign}(\hat{\gamma}))|,|\hat{\alpha}+\beta \operatorname{sign}(\hat{\gamma})|)) .
\end{gathered}
$$

4. To comply with goal $4, \hat{\alpha}$ should not be an integer multiple of $\beta$.

5. To comply with goal 5 without violating lower numbered goals,

$$
\hat{\alpha}=0 \vee(\operatorname{sign}(\hat{\alpha})=\operatorname{sign}(\beta \hat{\gamma}) \wedge|\hat{\gamma}|<1) \vee\left(\alpha \neq 0 \wedge \operatorname{sign}(\hat{\alpha}) \neq \operatorname{sign}(\beta \hat{\gamma}) \wedge|\hat{\gamma}| \leq \frac{1}{2}\right) .
$$

6. For compliance with goal 6 , every result entry for test family 1 should be identical to the entry 2 rows down and one column left, whereas every result entry for test family 2 should be identical to the entry 2 rows down and one column right. To equally assess the top two rows, the bottom two rows, the leftmost column and the rightmost column, I computed extra neighbors bordering those shown. When there were differences, I did not penalize the best displayed results for equivalent entries unless their was a better displayed result one column and one or two rows outside the table. However, I did penalize all of the not-best members for equivalent entries. I similarly tested the result in Table 16 against the equivalent expressions $\sqrt{w} / w$ and $w^{2} /\left(w^{2}\right)^{3 / 2}$. It is of course possible for an entry to pass these tests but fail for more widely separated equivalent inputs.

7. To test compliance with goal 7 , I resimplified each result with either default simplification or the optional transformation used for the original input, then checked for identical results. Compliance with this goal is not discernible from merely inspecting the result entries.

8. To test compliance with goal 8 , I manually rationalized the results having a fractional power in the denominator and $\alpha \neq 0$ by multiplying the numerator and denominator by $\left(w^{-2}\right)^{1 / 3}$ for test family 1 or $\sqrt{w^{2}}$ for test family 2 . It was counted as a flaw if and only if that forced rationalization did not introduce a removable singularity or increase its magnitude.

\subsection{Remarks about particular results.}

Maxima also has a relevant rat(...) function. For these examples, it generally produces the same result as default simplification, except that fractional powers are represented as an integer power of a reciprocal power - or an integer power of $\sqrt{\cdots}$ for half-integer powers. Thus a default result $w^{3}\left(w^{2}\right)^{5 / 3}$ would instead be $w^{3}\left(\left(w^{2}\right)^{1 / 3}\right)^{5}$, and a default

result $w^{3}\left(w^{2}\right)^{5 / 2}$ would instead be $w^{3}{\sqrt{w^{2}}}^{5}$. The standard definition of $u^{m / n}$ for reduced 
integers $m$ and $n$ is $\left(u^{1 / n}\right)^{m}$, which is consistent with the alternate definition $e^{\ln (u) m / n} 7$ Consequently, the Maxima rat (...) function makes the standard interpretation of the result more explicit at the expense of clutter. Nonetheless, it might be helpful as a precursor to semantically substituting a new expression for $\left(w^{2}\right)^{1 / 3}$ via syntactic substitution in a expression containing $\left(w^{2}\right)^{m / 3}$ for several different integer $m$. For the sake of brevity, results are not included for the rat (...) function because its flaws were very nearly identical to default simplification, regarding $\left(\left(w^{2}\right)^{1 / n}\right)^{m}$ as $\left(w^{2}\right)^{m / n}$.

Mathematica, Maxima and Maple also respectively have relevant PowerExpand[...], $\operatorname{radcan}(\ldots)$ and simplify (..., symbolic) functions. However, they always transform $\left(w^{\beta}\right)^{\gamma}$ to $w^{\beta \gamma}$, which is not equivalent along entire rays from $w=0$. I didn't test these functions because their purpose is presumably partly to allow these risky unconditional transactions for consenting adults. However, these three systems, Derive and TI computer algebra also have safe ways to enable such desired transformations, when justified, by declaring, for example, that certain variables are real or positive.

"... a man who thought he could somehow pull up the root without affecting the power." -adapted from Gilbert K. Chesterton

To make sure that $w$ is regarded as a complex variable and the principal branch is used rather than the real branch:

- All of the Derive results follow a prior declaration $w: \in$ Complex.

- All of the TI-CAS results necessarily used $w$ _ rather than $w$ to manifestly declare it as a complex indeterminate. However for consistency $w_{-}$is displayed in all of the tables as $w$ because Table 12 is shared with Maple for brevity.

- All of the Maxima results followed a prior assignment domain : complex and a prior declaration declare(w, complex).

As illustrated by Tables 15 through [17, the Maple simplify(...) function expresses halfinteger powers of squares or of reciprocals of squares using the Maple csgn(...) function defined by

$$
\operatorname{csgn}(w):= \begin{cases}1, & \text { if } \Re(w)>0 \vee \Re(w)=0 \wedge \Im(w) \geq 0, \\ -1, & \text { otherwise. }\end{cases}
$$

The right side of this definition is a simplified special instance of form 4 , for which $\operatorname{csgn}(w)$ is a convenient abbreviation for those familiar with it 8

Regarding Table 17.

\footnotetext{
${ }^{7}$ This is not generally equivalent to $\left(u^{m}\right)^{1 / n}$ : "be faithful to your roots" - Mason Cooley.

${ }^{8}$ Jeffrey [5] uses the unwinding function to generalize csgn to a $C_{n}$ that works for all fractional powers. If and when implemented in Maple, that will avoid unwelcome mixtures of $\operatorname{csgn}(.$.$) with other form 4$ notations for results containing both half-integer and other nested powers.
} 
- Mathematica (hence also the Appendix rewrite rules) did the automatic transformation

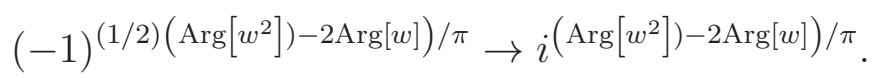

Although it eliminates the $1 / 2$ factor from the exponent in this case, it does so at the expense of candidness by introducing $i$ into an expression that is real for all $w$.

- For TI-CAS the arg function is spelled "angle" and regrettably angle $(0)$ is returned unchanged rather than transforming to 0 . Therefore the input was

$$
\frac{\sqrt{w_{-}^{2}}}{w_{-}}-(-1)^{\operatorname{when}\left(w_{-}=0,0,(1 / 2)\left(\operatorname{angle}\left(w_{-}{ }^{2}\right)-2 \operatorname{angle}\left(w_{-}\right)\right) / \pi\right)} \text {. }
$$

As indicated in Table 17, the real power of -1 in the input was changed to an imaginary power of $e$ in the result. This has the candidness disadvantage of introducing $i$ into an expression that is real for all real $w_{-}$.

- For Derive the arg function is spelled "phase" and regrettably phase(0) returns $\pi / 2 \pm \pi / 2$, which denotes an unknown element of $\{0, \pi\}$, which are the only two possibilities for real arguments. Therefore the input was

$$
\frac{\sqrt{w^{2}}}{w}-(-1)^{\operatorname{IF}\left(w=0,0,(1 / 2)\left(\operatorname{PHASE}\left(w^{2}\right)-2 \operatorname{PHASE}(w)\right) / \pi\right)}
$$

- For Maple, the arg function is spelled "argument", and for Maxima it is spelled "carg".

If you are interested in results for some other systems, then try a few of the examples that are heavily flawed for most of the five tested systems 9 First do whatever is necessary so that fractional powers use the principal branch and $w$ is regarded as a complex indeterminate.

\section{Four alternative forms}

Section 1 explains the reasons for four separate forms.

\subsection{Form 1: Reduction of outer fractional exponents to $(-1,1)$}

\section{Definition. For $x \in \mathbb{R}$ the integer part function}

$$
\operatorname{Ip}(x):= \begin{cases}\lfloor x\rfloor, & \text { if } x \geq 0 \\ \lceil x\rceil, & \text { otherwise. }\end{cases}
$$

\footnotetext{
${ }^{9}$ If you are familiar enough with those systems, then most of them probably have a quick way to generate all of the results for test families 1 and 2 by entries analogous to the following one for Mathematica:

$$
\text { Table }\left[\text { Table } \left[w^{j}\left(w^{2}\right)^{k},\{k,-7 / 3,8 / 3\},\{j,-3,3\}\right.\right. \text { //TableForm }
$$

I am interested in knowing your results.
} 
Definition. For $x \in \mathbb{R}$ the fractional part function $\operatorname{Fp}(x):=x-\operatorname{Ip}(x)$.

Proposition 1. For $\beta \in \mathbb{Q}, \gamma \in \mathbb{Q}-\mathbb{Z}$, and arbitrary expression $w \in \mathbb{C}$,

$$
\left(w^{\beta}\right)^{\gamma} \equiv w^{\beta \operatorname{Ip}(\gamma)}\left(w^{\beta}\right)^{\operatorname{Fp}(\gamma)} .
$$

Proof. We have

$$
\left(w^{\beta}\right)^{\gamma} \equiv\left(w^{\beta}\right)^{\operatorname{Ip}(\gamma)}\left(w^{\beta}\right)^{\mathrm{Fp}(\gamma)}
$$

because:

1. With $\gamma \in \mathbb{Q}-\mathbb{Z}, \operatorname{Ip}(\gamma)=0 \vee \operatorname{sign}(\operatorname{Ip}(\gamma))=\operatorname{sign}(\operatorname{Fp}(\gamma))$.

2. For any expression $u \in \mathbb{C}$ and $r_{1}, r_{2} \in \mathbb{Q} \mid r_{1}=0 \vee \operatorname{sign} r_{1}=\operatorname{sign} r_{2}$,

$$
u^{r_{1}+r_{2}} \equiv u^{r_{1}} u^{r_{2}},
$$

even at $u=0$ with $r_{1}$ and $r_{2}$ both negative, making both sides of (10) be complex infinity.

3. By Proposition 4 we also have $\left(w^{\beta}\right)^{\operatorname{Ip}(\gamma)} \equiv w^{\beta \operatorname{Ip}(\gamma)}$ because $\operatorname{Ip}(\gamma) \in \mathbb{Z}$.

Therefore Form 1 is simply to transform $w^{\alpha}\left(w^{\beta_{1}}\right)^{\gamma_{1}} \cdots\left(w^{\beta_{n}}\right)^{\gamma_{n}}$ toward canonicality by transforming every positive fraction $\gamma_{k}$ to the interval $(0,1)$ and every negative fraction $\gamma_{k}$ to the interval $(-1,0)$. The various $w^{\operatorname{Ip}\left(\gamma_{k}\right) \beta_{k}}$ are combined with the original $w^{\alpha}$, giving a transformed expression

$$
\widehat{W}:=w^{\hat{\alpha}}\left(w^{\beta_{1}}\right)^{\hat{\gamma}_{1}} \cdots\left(w^{\beta_{n}}\right)^{\hat{\gamma}_{n}}
$$

where $\hat{\alpha}$ might be 0 .

This form 1 satisfies goals 1 and 7, while possibly contributing progress toward goals $2,3,5$ and 6 . This form also has the advantage that if $\left(w^{\beta}\right)^{\hat{\gamma}}$ is subsequently raised to any power $\lambda$, then we can simplify it to the simplified value of $\left(w^{\beta}\right)^{\gamma \lambda}$ by Proposition 3 because $-1<\hat{\gamma}<1$. For example,

$$
\left(\left(w^{2}\right)^{3 / 4}\right)^{7 / 6} \rightarrow\left(w^{2}\right)^{7 / 8}
$$

Although there is no such thing as a free radical in computer algebra, this transformation of each nested power is fast and easy to implement because it occurs only for certain fractional powers of powers, which are relatively rare, and very little work is done even when it does occur. There is no good reason why default simplification shouldn't do at least this much.

However, default simplification for many systems unavoidably collects similar factors, resulting in a partial reversal of this transformation whenever a resulting unnested exponent $\hat{\alpha}$ is identical to one of the inner nested exponents. This happens for Derive, TI-CAS and Mathematica, but not for Maple or Maxima. With unavoidable collection, unconditional magnitude reduction of fractional outer exponents can lead to an infinite recursion such as

$$
w\left(w^{2}\right)^{5 / 3} \rightarrow w^{2}\left(w^{2}\right)^{2 / 3} \rightarrow w\left(w^{2}\right)^{5 / 3} \rightarrow \cdots .
$$

Therefore in the Appendix Mathematica rewrite rules: 
- Transformation $\left(w^{\beta}\right)^{\gamma} \rightarrow w^{\operatorname{Ip}(\gamma) \beta}\left(w^{\beta}\right)^{\operatorname{Fp}(\gamma)}$ is used unconditionally only prior to default simplification.

- The rewrite rules that are active during default and optional transformations do not reduce the magnitude of $\gamma$ if doing so would give an unnested exponent $\hat{\alpha}$ identical to $\beta$.

- This transformation is used after default simplification only if there is an unnested factor $w^{\alpha}$ and the transformation would not be reversed by unavoidable collection of similar powers.

Implementations for other systems might have to overcome this difficulty in some other way, or compromise and not always produce a form with outer fractional exponents in the interval $(-1,1)$ when $w^{\alpha}$ can't be fully absorbed into some nested power.

\subsection{Form 2: Further reducing some outer exponents to $(-1 / 2$, $1 / 2]$}

Form 2 is form 1 supplemented by an additional transformation.

Expression $\widehat{W}$ given by definition (11) is equivalent to expression $W$ everywhere that $W$ is defined, because at the only questionable point $w=0$ :

1. Expressions $W$ and $\widehat{W}$ are both 0 if $\alpha \geq 0$ and all of the $\beta_{k} \gamma_{k}$ are positive.

2. Otherwise expression $W$ and $\widehat{W}$ are both complex infinity if $\alpha \leq 0$ and all of the $\beta_{k} \gamma_{k}$ are negative.

3. Otherwise if $\hat{\alpha} \geq 0$ and all $\hat{\beta}_{k} \hat{\gamma}_{k}>0$, then $W$ is $0 / 0$ but $\widehat{W}$ has improved to 0 .

4. Otherwise if $\hat{\alpha} \leq 0$ and all $\hat{\beta}_{k} \hat{\gamma}_{k}<0$, then $W$ is $0 / 0$ but $\widehat{W}$ has improved to complex infinity.

5. Otherwise both $W$ and $\widehat{W}$ are $0 / 0$. However, the magnitude of the multiplicity of the removable singularity is less for $\widehat{W}$ if for any $\gamma_{k},\left|\gamma_{k}\right| \geq 1$.

Expression $\widehat{W}$ is canonical in cases 1 through 4 , but not necessarily for case 5 . For example,

1. The different equivalent expressions $z^{-1}\left(z^{2}\right)^{2 / 3}$ and $z\left(z^{2}\right)^{-1 / 3}$ both have outer exponents in $(-1,1)$. Of these two alternatives, the latter is preferable for most purposes because the $|-2 / 3|<|4 / 3|$, making multiplicity of the uncanceled portion of the removable singularity have a smaller magnitude. Thus a rationalized numerator is sometimes preferable to a rationalized denominator.

2. The different expressions $z^{-3}\left(z^{2}\right)^{1 / 2}$ and $z^{-1}\left(z^{2}\right)^{-1 / 2}$ both have outer exponents in $(-1,1)$, and they are equivalent wherever the first alternative is defined. However, the latter unrationalized denominator is preferable because the former is $0 / 0$ at $z=0$ where the latter is defined and equal to the complex infinity limit of the former. 
3. The different equivalent expressions $z\left(z^{2}\right)^{-1 / 2}$ and $z^{-1}\left(z^{2}\right)^{1 / 2}$ both have outer exponents in $(-1,1)$, and the multiplicities of the uncanceled portion of their removable singularity at $z=0$ are both 1 . Of these two alternatives, the latter is slightly preferable because it has a traditionally rationalized denominator rather than a rationalized numerator.

Thus after producing form 1 we can sometimes add 1 to a negative $\hat{\gamma}_{k}$ or subtract 1 from a positive $\hat{\gamma}_{k}$, then adjust $\alpha$ accordingly to reduce the magnitude of the overall removable singularity - perhaps entirely. If not, perhaps we can at least contribute toward goals 2 , 6 and 8 by rationalizing a square root in the denominator.

Let

$$
\begin{aligned}
\Delta_{k} & :=\beta_{k} \hat{\gamma}_{k}, \\
\Delta & :=\alpha+\Delta_{1}+\cdots+\Delta_{n} .
\end{aligned}
$$

Transforming any of the $\left(w^{\beta_{k}}\right)^{\gamma_{k}}$ to $w^{m_{k} \beta_{k}}\left(w^{\beta_{k}}\right)^{\gamma_{k}-m_{k}}$ for any integer $m_{k}$ leaves $\Delta$ unchanged.

Our primary goal is, whenever possible, to make all of the $\Delta_{k}$ have the same sign and for $\alpha$ to have either the same sign or be 0 . A secondary goal is to prefer $-1 / 2<\hat{\gamma}_{k} \leq 1 / 2$. Therefore, the algorithm to convert form 1 to form 2 is:

1. If $\Delta>0$, then for each $\Delta_{k}<0$, add $\operatorname{sign}\left(\beta_{k}\right)$ to $\hat{\gamma}_{k}$ and subtract $\left|\beta_{k}\right|$ from $\alpha$, then return the result.

2. If $\Delta<0$, then for each $\Delta_{k}>0$, subtract $\operatorname{sign}\left(\beta_{k}\right)$ from $\hat{\gamma}_{k}$ and add $\left|\beta_{k}\right|$ to $\alpha$, then return the result.

3. For each $\hat{\gamma}_{k}>1 / 2$, subtract 1 from $\hat{\gamma}_{k}$ and add $\beta_{k}$ to $\alpha$.

4. For each $\hat{\gamma} \leq-1 / 2$, add 1 to $\hat{\gamma}_{k}$ and subtract $\beta_{k}$ from $\alpha$.

5. Return the result.

This canonical form 2 satisfies all of the goals except for the aesthetic goal 4 .

For brevity the Appendix rewrite rules consider only one $\Delta_{k}$ at a time. This is sufficient for all of the test cases, which have only one nested power. For an industrialstrength implementation, each time we multiply a fractional power of a power by a product of one or more factors, we should inspect those factors for identical expressions $w$ and apply the above algorithm if that subset if non-empty. The cost is $O\left(n_{c}\right)$ where $n_{c}$ is the number of cofactors. The opportunity occurs only when multiplying a fractional power of a power, which is rare; and the number of factors in a product is typically quite small. Therefore it also quite reasonable to do this in default simplification.

\subsection{Form 3: Finally, fully absorb $w^{\alpha}$ into a fractional power if possible}

Form 3 is form 2 followed by an additional transformation. 
Form 2 can result in an expression such as $z^{4}\left(z^{2}\right)^{1 / 2}$, for which many users would regard $\left(z^{2}\right)^{5 / 2}$ as a simpler result because it has one less factor. We can often absorb at least some of $z^{\alpha}$ into one of the $\left(z^{\beta_{k}}\right)^{\gamma_{k}}$ by the transformation

$$
z^{\alpha}\left(z^{\beta_{k}}\right)^{\gamma_{k}} \rightarrow z^{\beta_{k} \operatorname{Fp}\left(\alpha / \beta_{k}\right)}\left(z^{\beta_{\mathbf{k}}}\right)^{\gamma_{k}+\operatorname{Ip}\left(\alpha / \beta_{k}\right)},
$$

which doesn't change the domain of definition. However, this transformation seems inadvisable unless $\operatorname{Fp}\left(\alpha / \beta_{k}\right)=0$, because otherwise it increases the contribution of a troublesome nested power without reducing the number of factors. Also, this transformation is problematic during intermediate computations even if $\operatorname{Fp}\left(\alpha / \beta_{k}\right)=0$, because when there is more than one nested power, then more than one might be eligible, making it awkward to maintain canonicality achieved by form 2. Moreover, absorption conflicts with transformations done to obtain form 1 or 2 , thus risking infinite recursion.

A solution to this dilemma is to fully absorb $w^{\alpha}$ only just before display - after all other default and optional simplification. This does have the minor disadvantage that what the user sees doesn't faithfully represent the internal representation. However, that bridge has already been crossed by most systems, which for speed and implementation simplicity internally use, for example, $(\ldots)^{1 / 2}$ to represent a displayed $\sqrt{\cdots}$ and $a+-1 * b$ to represent a displayed $a-b$.

When there is more than one nested power of $w$, then there might be more than one way to absorb $\alpha$ completely into those nested powers. For example,

$$
\begin{aligned}
w^{6}\left(w^{2}\right)^{1 / 2}\left(w^{3}\right)^{1 / 2}\left(w^{4}\right)^{1 / 2} & \equiv\left(w^{2}\right)^{\mathbf{7} \mathbf{2}}\left(w^{3}\right)^{1 / 2}\left(w^{4}\right)^{1 / 2} \\
& \equiv\left(w^{2}\right)^{1 / 2}\left(w^{3}\right)^{\mathbf{5} / \mathbf{2}}\left(w^{4}\right)^{1 / 2} \\
& \equiv\left(w^{2}\right)^{\mathbf{3 / 2}}\left(w^{3}\right)^{1 / 2}\left(w^{4}\right)^{\mathbf{3} \mathbf{2}}
\end{aligned}
$$

In general, the possible resulting expressions are given by

$$
\left(w^{\beta_{1}}\right)^{\gamma_{1}+m_{1}}\left(w^{\beta_{2}}\right)^{\gamma_{2}+m_{2}} \cdots\left(w^{\beta_{n}}\right)^{\gamma_{n}+m_{n}},
$$

where the tuple of integers $\left\langle m_{1}, m_{2}, \ldots, m_{n}\right\rangle$ is a solution to the linear Diophantine equation

$$
m_{1} \beta_{1}+m_{2} \beta_{2}+\cdots+m_{n} \beta_{n}=\alpha .
$$

Solutions exist if and only if $\alpha$ is an integer multiple of $\operatorname{gcd}\left(\beta_{1}, \beta_{2}, \ldots \beta_{n}\right)$, in which case there might be a countably infinite number of tuples. However, to avoid introducing removable singularities or increasing the magnitude of their multiplicity, we are only interested in solutions for which $\operatorname{sign}\left(m_{j} \beta_{j}\right) \equiv \operatorname{sign}(\alpha)$ for $j=1,2, \ldots, n$. Papp and Vizvari [7] describe an algorithm for solving such sign-constrained linear Diaphantine equations, and the Mathematica Reduce [...] function can solve such equations. For example, suppose our canonical form 2 result is

$$
z^{14}\left(z^{6 / 7}\right)^{1 / 2}\left(z^{10 / 7}\right)^{1 / 3}
$$


In Mathematica, we can determine the family of integers $m_{1} \geq 0$ to add to $1 / 2$ and $m_{2} \geq 0$ to add to $1 / 3$ that together absorb $z^{14}$ as follows:

$$
\begin{gathered}
\ln [1]:=\text { Reduce }\left[\frac{6}{7} m_{1}+\frac{10}{7} m_{2}==14 \& \& \frac{6}{7} m_{1} \geq 0 \& \& \frac{10}{7} m_{2} \geq 0,\left\{\mathrm{~m}_{1}, \mathrm{~m}_{2}\right\}, \text { Integers }\right] \\
/ / \text { TraditionalForm }
\end{gathered}
$$

Out $[1] / /$ TraditionalForm $=\left(m_{1}=3 \wedge m_{2}=8\right) \vee\left(m_{1}=8 \wedge m_{2}=5\right) \vee\left(m_{1}=13 \wedge m_{2}=2\right)$

Regarding the choice between alternative absorptions, canonicality is not as important for a final displayed result as it is during intermediate calculations where it facilitates important cancellations. However, with more than one solution, we could choose one in a canonical way as follows: Order the $\beta_{j}$ in some canonical way, such as the way they order in $\left(w^{\beta_{1}}\right)^{\gamma_{1}} \cdots\left(w^{\beta_{n}}\right)^{\gamma_{n}}$, then to choose the solution for which $m_{1}$ is smallest, with ties broken according to which $m_{2}$ is smallest, etc.

Solution of sign-constrained linear Diophantine equations can be costly - probably too costly for default simplification. Consequently, the rewrite rules in the Appendix simply absorb $w^{\alpha}$ if and only if it can be completely absorbed into a single power of a power, in which case the particular one is the first one encountered by the pattern matcher. This is canonical, but it doesn't absorb $w^{\alpha}$ for examples such as (13). However, this transformation is inexpensive because it is done only once in one pass over the expression just prior to display, and the transformation requires comparing a power of a power with its cofactors only in products where powers of powers occur.

All but this absorption rule are automatically applied before default simplification so that, for example, the input

$$
\frac{w-w}{\frac{\sqrt{z^{2}}}{z^{3}}-\frac{1}{z \sqrt{z^{2}}}}
$$

correctly simplifies to indeterminate, meaning $0 / 0$, rather than to 0 .

The rewrite rules in the Appendix are not much more than the minimal amount necessary to generate the form 3 results in Tables 1 and 8 , together with the relevant rows in Tables 16 and 17.

\subsection{Form 4: One unnested power times a unit-magnitude factor}

Form 4 is quite different from forms 1 through 3.

A universal principal-branch formula for transforming a nested power to an unnested power is

$$
\left(w^{\beta}\right)^{\gamma} \rightarrow(-1)^{\tau} w^{\beta \gamma}
$$

where

$$
\tau:= \begin{cases}0, & \text { if } \arg (0)=0, \\ \frac{\gamma\left(\arg \left(w^{\beta}\right)-\beta \arg (w)\right)}{\pi}, & \text { otherwise, }\end{cases}
$$

with short-circuit evaluation so that the "otherwise" result expression is not evaluated when the "if" test is true. 
The transformation given by formulas (14) and (15) can be derived from the identities

$$
\begin{aligned}
|p| & \equiv(-1)^{-\arg (p) / \pi} p \quad \text { for } p \neq 0, \\
\left|q^{\alpha}\right|^{\beta} & \equiv|q|^{\alpha \beta} .
\end{aligned}
$$

Notice that the unit-polar factor $(-1)^{\tau}$ is unit magnitude because $\arg (\ldots)$ is always real, as are the rational numbers $\gamma$ and $\beta$. Moreover, $(-1)^{\tau}$ is piecewise constant with pie-shaped pieces emanating from $w=0$ because $\arg \left(w^{\beta}\right)$ and $\beta \arg (w)$ have the same derivative with respect to $w$ everywhere they are both continuous, and each of them has a finite number of discontinuities.

An imaginary exponential $e^{i \pi \tau}$ is an alternative to $(-1)^{\tau}$, but it has the candidness disadvantage of introducing $i$ into a factor that can be real and always is for the common case where the outer exponent $\gamma$ is a half-integer.

If $\arg (0)$ is defined as 0 , as it is in Mathematica, Maple, and Maxima, then we can define $\tau$ more concisely and unconditionally as

$$
\tau:=\frac{\gamma\left(\arg \left(w^{\beta}\right)-\beta \arg (w)\right)}{\pi} .
$$

Proposition 2. If $w \geq 0$, then $\tau=0$.

Proof. When $w=0, \tau=0$ follows immediately from expression (15), and $w>0 \Rightarrow \arg \left(w^{\beta}\right)=0 \wedge \arg (w)=0 \Rightarrow \gamma\left(\arg \left(w^{\beta}\right)-\beta \arg (w)\right) / \pi=0 \Rightarrow \tau=0$.

Proposition 3. If $-1<\beta \leq 1$, then $\tau=0$.

Proof. $-1<\beta \leq 1 \Rightarrow \arg \left(w^{\beta}\right)=\beta \arg (w) \Rightarrow \gamma\left(\arg \left(w^{\beta}\right)-\beta \arg (w)\right) / \pi=0 \Rightarrow \tau=$ 0 .

Proposition 4. If $\gamma$ is integer, then $(-1)^{\tau}=1$.

Proof. $\arg \left(w^{\beta}\right)$ is $\beta \arg (w)$ plus an even integer multiple of $2 \pi$. Thus when $\gamma$ is an integer, then $\gamma\left(\arg \left(w^{\beta}\right)-\beta \arg (w)\right) / \pi$ is an even integer, making $\tau$ be an even integer, making $(-1)^{\tau}=1$.

The simplification afforded by these three propositions should have already been exploited with bottom-up default simplification, in which case $\left(w^{\beta}\right)^{\gamma}$ will have already been simplified to $w^{\beta \gamma}$. If it isn't, then that is another opportunity to improve the system for very little effort 10 Thus, because $\beta$ and $\gamma$ are explicit non-zero rational numbers, without loss of generality this article assumes that $w$ isn't known to be nonnegative, and that $\beta \leq-1$ or $\beta>1$, and that $\gamma$ is non-integer.

Using transformation (14) on every $\left(w^{\beta_{k}}\right)^{\gamma_{k}}$ in $W$ defined by (11) then collecting powers of -1 gives

$$
\bar{W}=(-1)^{\sigma} w^{\alpha+\beta_{1} \gamma_{1}+\cdots+\beta_{n} \gamma_{n}},
$$

where $\sigma$ is a simplified sum of terms of the form (15) or (18).

The factor $(-1)^{\sigma}$ is also unit magnitude with pie-shaped piecewise constant pieces because it is the product of such factors. This form has two great advantages over the other three forms:

\footnotetext{
${ }^{10}$ Do your computer algebra system's default and optional transformations de-nest $\left(w^{\beta}\right)^{\gamma}$ for such $\beta$, $\gamma$, and $w$ declared non-negative?
} 
- All of the exponents have been combined into a single unnested exponent.

- Cancelable singularities are always completely canceled.

Unfortunately this comes at the expense of a form that is usually bulkier than the other forms

Simplification of individual piecewise expressions and combinations of such expressions is currently rather weak in most systems, but Carette 2] describes a canonical form for such expressions, so we can hope for improvement. In our case the piecewise expressions all have the same tests. Therefore we can add all of the 0s together and add all of the expressions involving $\arg (. .$.$) together into a single piecewise function. For example,$

$$
\begin{aligned}
& \frac{\left(z^{2}\right)^{3 / 2}\left(z^{3}\right)^{4 / 3}}{z^{6}}
\end{aligned}
$$

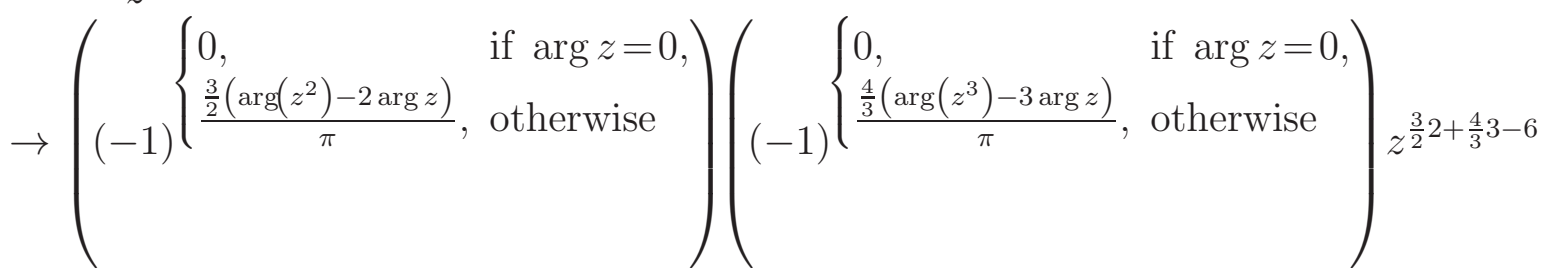

$$
\begin{aligned}
& \rightarrow\left(\begin{array}{ll}
0, & \text { if } \arg z=0 \\
\frac{\frac{3}{2} \arg \left(z^{2}\right)+\frac{4}{3} \arg \left(z^{3}\right)-7 \arg z}{\pi}, & \text { otherwise }
\end{array}\right) z
\end{aligned}
$$

If $\arg (0) \rightarrow 0$, then simplification of piecewise expressions isn't an issue here and the resulting exponent of -1 is simply $\left(\frac{3}{2} \arg \left(z^{2}\right)+\frac{4}{3} \arg \left(z^{3}\right)-7 \arg z\right) / \pi$. However, the result is not canonical either way, because starting with the equivalent canonical form 2 ,

$$
\frac{\sqrt{z^{2}}\left(z^{3}\right)^{1 / 3}}{z} \rightarrow\left(\begin{array}{ll}
0, & \text { if } \arg z=0 \\
\frac{\frac{1}{2} \arg \left(z^{2}\right)+\frac{1}{3} \arg \left(z^{3}\right)-2 \arg z}{\pi} & \text { otherwise }
\end{array}\right) z
$$

which has smaller magnitude coefficients. Thus for canonicality we could precede this transformation with a transformation to form 2. Equivalently we can adjust the coefficients of the $\arg \left(w^{\beta_{k}}\right)$ and $\arg (w)$ analogous to how we adjusted exponents to arrive at form 2. This is preferable because it also canonicalizes expressions of form 4 that are entered directly or generated by the system.

With pie-shaped pieces, $(-1)^{\sigma}$ can always be expressed in the more candid canonical form

$$
\begin{cases}c_{1}, & \text { if }-\pi<\arg w:: \theta_{1}, \\ c_{2}, & \text { if } \theta_{1}:: \arg w:: \theta_{2}, \\ \cdots & \cdots \\ c_{m}, & \text { otherwise, }\end{cases}
$$


where $c_{1}$ through $c_{m}$ are unit-magnitude complex constants, $\theta_{1}$ through $\theta_{m-1}$ are real constants in $(-\pi, \pi)$, and each instance of "::" is either " $<$ " or " $\leq$ " 11 Moreover:

1. When $w$ is real, then the positive and negative real axes are each entirely within one pie slice, enabling us to simplify $(-1)^{\sigma}$ to one unconditional constant or piecewise expression of the form

$$
\begin{cases}c_{1}, & \text { if } w:: 0, \\ c_{2} & \text { otherwise, }\end{cases}
$$

where "::" is one of the comparison operators " $>$ ", " $\geq$ ", $=$, " $\leq$ ", " $<$ ", or " $\neq$ ".

2. For half-integers or quarter-integer fractional powers, $(-1)^{\tau}$ can be expressed as a piecewise expression depending on the real and imaginary parts of $w$ rather than $\arg (w)$. For example,

$$
\begin{aligned}
& \frac{\left(w^{2}\right)^{1 / 2}}{w} \rightarrow \begin{cases}1 & \text { if } \Re(w)>0 \vee \Re(w) \geq 0 \wedge \Im(w) \geq 0, \\
-1 & \text { otherwise; }\end{cases} \\
& \frac{\left(w^{4}\right)^{1 / 4}}{w} \rightarrow \begin{cases}1 & -\Re(w)<\Im(w) \leq \Re(w), \\
-i & \text { if }-\Im(w)<\Re(w) \leq \Im(w), \\
-1 & \Re(w)<\Im(w) \leq-\Re(w), \\
i & \text { otherwise. }\end{cases}
\end{aligned}
$$

Notice that the right side of result (22) is the definition of the Maple csgn function.

Without an abbreviation such as $\operatorname{csgn}(. .$.$) , Most implementers will probably want to$ avoid form 4 as a default even when $\arg (0) \rightarrow 0$, because $(-1)^{\sigma}$ is likely to be rather complicated nonetheless:

1. It will probably contain complicated square roots and arctangents if the real and imaginary parts of $w$ are given as exact numbers.

2. It will probably also contain piecewise sign tests if given real and imaginary parts that are non-numeric, such as for $w=x+i y$ with non-numeric real indeterminates $x$ and $y$.

3. It will probably contain radicals nested at least one deep if arg $(w)$ is a simple enough rational multiple of $\pi$.

4. Otherwise it will contain perhaps bulky sub-expressions $\arg (w)$ and $\arg \left(w^{\beta}\right)$ - or, worse yet, expressions involving square roots, arctangents, piecewise sign tests, and sub-expressions of the form $\Re(w)$ and $\Im(w)$.

As espoused by Corless and Jeffrey [4], expression $\tau$ can alternatively be defined in terms of the unwinding function $\kappa$ as:

$$
\tau:=2 \gamma \kappa(\beta \ln w) .
$$

\footnotetext{
${ }^{11}$ In a degenerate case, one or more of the pieces of pie might be a ray - very dietetic.
} 
This is more concise than definition (15), but a function that computes unwinding numbers isn't currently available externally in most computer algebra systems. Also, unless the system automatically transforms $\ln 0$ to $-\infty$, as is done in Mathematica and Derive, then definition (24) has the same disadvantages as using $\arg (\ldots) 12$

\subsection{Simplifying mixtures of form 4 with form 1,2 or 3}

If an expression contains a mixture of forms, then we should unify the forms to facilitate collection and cancelation. For example with $\arg (0) \rightarrow 0$, the three expressions

$$
\begin{gathered}
\frac{\left(z^{2}\right)^{1 / 2}}{z}, \\
(-1)^{\left(\arg \left(z^{2}\right) / 2-\arg z\right) / \pi}, \\
\begin{cases}1 & \text { if } \Re(w)>0 \vee \Re(w) \geq 0 \wedge \Im(w) \geq 0, \\
-1 & \text { otherwise }\end{cases}
\end{gathered}
$$

are equivalent. Therefore the result of any linear combination of them should transform either to 0 or a multiple of one of them. The rewrite rules in the Appendix don't address this issue.

In general it is easy to transform form (25) to form (26), which is only slightly more difficult to transform to either form (25) or form (27).

\section{$6 \quad$ Unimplemented extensions}

\subsection{More semantic pattern matching for $w$}

The Mathematica pattern matcher is mostly syntactic rather than semantic, and the rules in the Appendix do almost no transformation of the radicand expressions $w$ or any cofactors thereof. Thus recognition of opportunities relies mostly on the default transformations together with any optional transformations done by the user. Consequently, opportunities for the rules to simplify nested power products might not be recognized for radicands that aren't indeterminates. The rules work for most functional forms that have syntactically identical forms for the different instances of $w$, such as

$$
\frac{\left(\log \left[x^{2}(x+y)\right]^{2}\right)^{5 / 3}}{\log \left[x^{2}(x+y)\right]} \rightarrow \log \left[x^{2}(x+y)\right]\left(\log \left[x^{2}(x+y)\right]^{2}\right)^{2 / 3} .
$$

However the rules don't apply to all such functional form opportunities. For example,

$$
\frac{\left(\operatorname{Cos}[\theta]^{2}\right)^{5 / 3}}{\operatorname{Cos}[\theta]} \rightarrow\left(\operatorname{Cos}[\theta]^{2}\right)^{5 / 3} \operatorname{Sec}[\theta]
$$

\footnotetext{
${ }^{12}$ For TI-CAS, $\ln (0) \rightarrow$ undef. An error is inconveniently thrown by Maple for $\ln (0)$ and by Maxima for $\log (0)$.
} 
because default simplification transforms $\operatorname{Cos}[\theta]^{-1}$ to $\operatorname{Sec}[\theta]$.

Even more opportunities are unrecognized when $w$ is a sum. As an example of how to overcome this, the Appendix includes one extra rule that square-free factors radicands that are sums so that, for example,

$$
\frac{\left(z^{2}+2 z+1\right)^{5 / 3}}{z+1} \rightarrow \frac{\left((z+1)^{2}\right)^{5 / 3}}{z+1} \rightarrow(z+1)\left((z+1)^{2}\right)^{2 / 3} .
$$

Factored over the integers or square-free factored form is a good choice for radicands for other reasons too, and these forms are canonical when the radicand is a rational expression. However,

$$
\left(z^{2}+2 z+1\right)\left((z+1)^{2}\right)^{5 / 3} \rightarrow(z+1)^{2}\left((z+1)^{2}\right)^{5 / 3} \rightarrow\left((z+1)^{2}\right)^{8 / 3},
$$

would require another rule that factors the cofactor of a power of a power of a sum. Then, perhaps we would want another rule to factor sums containing such radicands so that

$$
z^{2}\left((z+1)^{2}\right)^{5 / 3}+2 z\left((z+1)^{2}\right)^{5 / 3}+\left((z+1)^{2}\right)^{5 / 3} \rightarrow\left((z+1)^{2}\right)^{8 / 3} .
$$

It is impossible to implement equivalence recognition for all possible expressions $w$ representable in general purpose systems, but it is worth expending a modest amount of execution time for default simplification and more time for optional transformations.

The Appendix leaves most such opportunities unimplemented because the simplifications described here are so fundamental and low level that they should be part of the built-in transformations. Good simplification of nested power products is more appropriately built into a system rather than provided as an optionally loaded package that most users are unlikely to know about and load into every session. So rather than implementing a comprehensive package for one system, the intent of this article is to inspire implementers of all systems to improve some very fundamental transformations - at least to the extent that it can be done economically.

\subsection{Non numeric exponents}

Although not implemented in the rules of the Appendix, more generally the exponents for forms 1 through 4 can be Gaussian fractions or even symbolic, in which case we can still apply these transformations to the rational numeric parts of the exponents. For example,

$w^{3 \xi+\rho}\left(w^{\xi}\right)^{3 / 2+\omega \pi i} \rightarrow\left(w^{\xi}\right)^{3} w^{\rho}\left(w^{\xi}\right)^{1+1 / 2+\omega \pi i} \rightarrow\left(w^{\xi}\right)^{4} w^{\rho}\left(w^{\xi}\right)^{1 / 2+\omega \pi i} \rightarrow w^{4 \xi+\rho}\left(w^{\xi}\right)^{1 / 2+\omega \pi i}$.

As another example, if a user has declared the variable $n$ to be integer, then

$$
w^{-n}\left(w^{2}\right)^{n+1 / 2} \rightarrow w^{n}\left(w^{2}\right)^{1 / 2} .
$$

To some extent, the methods can also be extended to handle floating-point and symbolic real expressions for exponents $\alpha$ and $\beta_{k}$. For example,

$$
\begin{aligned}
w^{4.321}\left(w^{1.234}\right)^{3 / 2} & \rightarrow w^{5.555} \sqrt{w^{1.234}} \\
w^{2-\pi}\left(w^{\pi}\right)^{3 / 2} & \rightarrow w^{2} \sqrt{w^{\pi}} .
\end{aligned}
$$




\section{Summary}

This article:

1. shows that many widely-used computer algebra systems have significant room for improvement at simplifying sub-expressions of the form $w^{\alpha}\left(w^{\beta_{1}}\right)^{\gamma_{1}} \cdots\left(w^{\beta_{n}}\right)^{\gamma_{n}}$;

2. defines four different simplified forms with good properties;

3. explains how to compute these forms;

4. includes a demonstration implementation of form 3 via Mathematica rewrite rules.

\section{Acknowledgment}

I thank Sam Blake for his helpful assistance with Mathematica, Daniel Lichtblau for information about the algorithm in Reduce [...], and a referee for many fine suggestions.

\section{References}

[1] Brown, W.S: On computing with factored rational expressions. Proceedings of EUROSAM '74, ACM SIGSAM Bulletin 8 (3), pp. 26-34, 1974.

[2] Carette, J., A canonical form for piecewise defined functions, Proceedings of ISSAC 2007, pp. 77-84.

[3] Corless, R.M., Jeffrey, D.J., Well ... It isn't quite that simple. ACM SIGSAM Bulletin 26 (3), pp. 2-6, 1992.

[4] Corless, R.M. and Jeffrey, D.J., Editor's corner: The unwinding number, ACM Communications in Computer Algebra 30 (2), pp. 28-35, 1996.

[5] Jeffrey, D.J., Branching out with inverse functions, 2009, http://www . activemath.org/workshops/MathUI/09/proc/

[6] Moses, J: Algebraic simplification, a guide for the perplexed. Proceedings of the second ACM symposium on symbolic and algebraic manipulation, pp. 282-304, 1971

[7] Papp, D. and Vizvari, B: Effective solution of linear Diophantine equation systems with an application to chemistry, Journal of Mathematical Chemistry 39 (1), pp. 15-31, 2006.

[8] Rich, A.D. and Jeffrey, D.J., Function evaluation on branch cuts, Communications in Computer Algebra 30 (2), pp. 25-27, 1996.

[9] Stoutemyer, D.R., Useful computations need useful numbers, ACM Communications in Computer Algebra 41 (3), pp. 75-99, 2007.

[10] Stoutemyer, D.R., Ten commandments for good default expression simplification, Journal of Symbolic Computation, 46 (7), pp. 859-887, 2011. 


\section{Appendix: Mathematica rewrite rules for $w^{\alpha}\left(w^{\beta_{1}}\right)^{\gamma_{1}} \cdots\left(w^{\beta_{n}}\right)^{\gamma_{n}}$}

(* EXTRA SIMPLIFICATION DONE BEFORE ORDINARY EVALUATION: *)

PreProductOfPowersOfPowers [(w_Plus) -(g_Rational /; ! IntegerQ[g])] := $\mathrm{Block}[\{$ squareFree $=$ FactorSquareFree $[\mathrm{w}]\}$, squareFree^g /; Head[squareFree] =!= Plus];

PreProduct0fPowers0fPowers $\left[\left(\mathrm{w}_{-}{ }^{-} \mathrm{b}_{-}\right)\right.$- (g_Rational /; $\left.\left.\mathrm{g}<=-1|| \mathrm{g}>=1\right)\right]:=$ $\mathrm{w}^{\wedge}($ IntegerPart $[\mathrm{g}] * \mathrm{~b}) *\left(\mathrm{w}^{\wedge} \mathrm{b}\right) \wedge$ FractionalPart $[\mathrm{g}]$;

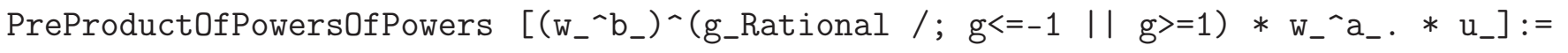
PreProductOfPowers0fPowers [w^(a+IntegerPart $[\mathrm{g}] * \mathrm{~b}) *\left(\mathrm{w}^{\wedge} \mathrm{b}\right) \wedge$ FractionalPart $\left.[\mathrm{g}] * \mathrm{u}\right]$;

PreProduct0fPowers0fPowers $\left[\left(\mathrm{w}_{-}{ }^{\wedge} \mathrm{b}_{-}\right){ }^{\wedge} \mathrm{g}_{-} * \mathrm{w}_{-}{ }^{\wedge} \mathrm{a}_{-} \cdot * \mathrm{u}_{-} \cdot /\right.$; Sign $[\mathrm{a}] \mathrm{!}=\mathrm{Sign}[\mathrm{b} * \mathrm{~g}]$ \&\& (Sign $[\mathrm{a}+\mathrm{b} * \operatorname{Sign}[\mathrm{g}]]==\operatorname{Sign}[\mathrm{b} *(\mathrm{~g}-\mathrm{Sign}[\mathrm{g}])]$ ।

$\operatorname{Min}[\operatorname{Abs}[\mathrm{a}], \operatorname{Abs}[\mathrm{b} * \mathrm{~g}]]$ > Min [Abs [a+b*Sign[g]], Abs [b*(g-Sign[g])]] ।

$\mathrm{g}==-1 / 2$ \&\& Min [Abs $[\mathrm{a}]$, Abs $[\mathrm{b} / 2]]==\operatorname{Min}[\operatorname{Abs}[\mathrm{a}-\mathrm{b}], \operatorname{Abs}[\mathrm{b} / 2]])]:=$ $\mathrm{w}^{\wedge}(\mathrm{a}+\mathrm{b} * \operatorname{Sign}[\mathrm{g}]) *\left(\mathrm{w}^{\wedge} \mathrm{b}\right) \wedge(\mathrm{g}-\operatorname{Sign}[\mathrm{g}]) * \mathrm{u}$;

PreProductOfPowersOfPowers [f_[args__] $:=$ Apply [f, Map [PreProductOfPowersOfPowers, \{args\}]];

PreProductOfPowersOfPowers [anythingElse_] := anythingElse;

(* EXTRA SIMPLIFICATION DURING ORDINARY EVALUATION: *)

Unprotect [Times];

$\left(\mathrm{w}_{-}{ }^{-} \mathrm{b}_{-}\right) \wedge \mathrm{g}_{-} * \mathrm{w}_{-}{ }^{-a_{-}} \cdot * \mathrm{u}_{-} \cdot / \operatorname{Sign}[\mathrm{a}] \quad \mathrm{S}=\operatorname{Sign}[\mathrm{b} * \mathrm{~g}]$ \&\&

(Sign $[a+b * \operatorname{Sign}[g]]==\operatorname{Sign}[b *(g-\operatorname{Sign}[g])]||$

$\operatorname{Min}[\operatorname{Abs}[\mathrm{a}], \operatorname{Abs}[\mathrm{b} * \mathrm{~g}]]>\operatorname{Min}[\operatorname{Abs}[\mathrm{a}+\mathrm{b} * \operatorname{Sign}[\mathrm{g}]], \operatorname{Abs}[\mathrm{b} *(\mathrm{~g}-\operatorname{Sign}[\mathrm{g}])]]$ ।।

$\operatorname{Min}[\operatorname{Abs}[\mathrm{a}], \operatorname{Abs}[\mathrm{b} * g]]==\operatorname{Min}[\operatorname{Abs}[\mathrm{a}+\mathrm{b} * \operatorname{Sign}[\mathrm{g}]]$, Abs [b*(g-Sign $[\mathrm{g}])]]$ \&\& $\operatorname{Abs}[\mathrm{g}]>\operatorname{Abs}[\mathrm{g}-\mathrm{Sign}[\mathrm{g}]] \mid \mathrm{|}$

$\mathrm{g}==-1 / 2 \& \& \operatorname{Min}[\operatorname{Abs}[\mathrm{a}], \operatorname{Abs}[\mathrm{b} / 2]]==\operatorname{Min}[\operatorname{Abs}[\mathrm{a}-\mathrm{b}], \operatorname{Abs}[\mathrm{b} / 2]]):=$ $\mathrm{w}^{\wedge}(\mathrm{a}+\mathrm{b} * \operatorname{Sign}[\mathrm{g}]) *\left(\mathrm{w}^{\wedge} \mathrm{b}\right) \wedge(\mathrm{g}-\operatorname{Sign}[\mathrm{g}]) * \mathrm{u}$;

$\left(w_{-}{ }^{-b} 1_{-}\right)^{\wedge} g 1_{-} *\left(w_{-}{ }^{\natural} b 2_{-}\right) \wedge g 2_{-} * u_{-} \cdot /$; Sign [b1*g1] != Sign[b2*g2] \&\&

Abs [b2] > Abs[b1] \&\& Sign [b2*(g2-Sign[g2])] == Sign [b1*g1 + b2*Sign[g2]]:= $\left(\mathrm{w}^{\wedge} \mathrm{b} 2\right)^{\wedge}(\mathrm{g} 2-\operatorname{Sign}[\mathrm{g} 2]) *\left(\left(\mathrm{w}^{\wedge}(\mathrm{b} 2 * \operatorname{Sign}[\mathrm{g} 2]) *\left(\mathrm{w}^{\wedge} \mathrm{b} 1\right)^{\wedge} \mathrm{g} 1\right) * \mathrm{u}\right)$;

Protect [Times];

(* EXTRA SIMPLIFICATION DONE AFTER ORDINARY EVALUATION: *) 


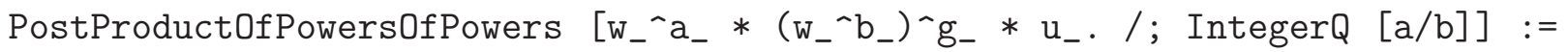
PostProductOfPowers0fPowers $\left[\left(w^{\wedge} b\right)^{\wedge}(g+a / b) * u\right]$;

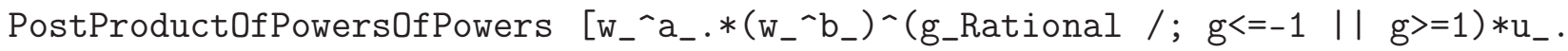

$/$; IntegerQ $[(\mathrm{a}+\mathrm{b} *$ IntegerPart $[\mathrm{g}]) / \mathrm{b}]]:=$

PostProductOfPowers0fPowers $\left[\left(\mathrm{u} * \mathrm{w}^{\wedge}(\mathrm{a}+\mathrm{b} *\right.\right.$ IntegerPart $\left.[\mathrm{b}])\right) *\left(\mathrm{w}^{\wedge} \mathrm{b}\right) \wedge$ FractionalPart $\left.[\mathrm{g}]\right]$;

PostProductOfPowersOfPowers [f_[args__] $]:=$

Apply [f, Map [PostProductOfPowersOfPowers, \{args\}]];

PostProductOfPowersOfPowers [anythingElse_] := anythingElse;

\$Post $=$ PostProduct0fPowers0fPowers; $\quad$ \$Pre = PreProductOfPowersOfPowers;

\section{Tables 1 through 17}

Table 1: Unflawed results of Appendix rewrite rules for 1 st row $\times 1$ st column. Compare with Tables 2 through 7

\begin{tabular}{|c||c|c|c|c|c|c|}
\hline$\vec{\downarrow}$ & $\left(w^{2}\right)^{-7 / 3}$ & $\left(w^{2}\right)^{-4 / 3}$ & $\left(w^{2}\right)^{-1 / 3}$ & $\left(w^{2}\right)^{2 / 3}$ & $\left(w^{2}\right)^{5 / 3}$ & $\left(w^{2}\right)^{8 / 3}$ \\
\hline \hline$w^{-3}$ & $\frac{1}{w^{7}\left(w^{2}\right)^{1 / 3}}$ & $\frac{1}{w^{5}\left(w^{2}\right)^{1 / 3}}$ & $\frac{1}{w^{3}\left(w^{2}\right)^{1 / 3}}$ & $\frac{1}{w\left(w^{2}\right)^{1 / 3}}$ & $\frac{w}{\left(w^{2}\right)^{1 / 3}}$ & $w\left(w^{2}\right)^{2 / 3}$ \\
\hline$w^{-2}$ & $\frac{1}{\left(w^{2}\right)^{10 / 3}}$ & $\frac{1}{\left(w^{2}\right)^{7 / 3}}$ & $\frac{1}{\left(w^{2}\right)^{4 / 3}}$ & $\frac{1}{\left(w^{2}\right)^{1 / 3}}$ & $\left(w^{2}\right)^{2 / 3}$ & $\left(w^{2}\right)^{5 / 3}$ \\
\hline$w^{-1}$ & $\frac{1}{w^{5}\left(w^{2}\right)^{1 / 3}}$ & $\frac{1}{w^{3}\left(w^{2}\right)^{1 / 3}}$ & $\frac{1}{w\left(w^{2}\right)^{1 / 3}}$ & $\frac{w}{\left(w^{2}\right)^{1 / 3}}$ & $w\left(w^{2}\right)^{2 / 3}$ & $w^{3}\left(w^{2}\right)^{2 / 3}$ \\
\hline$w^{0}$ & $\frac{1}{\left(w^{2}\right)^{7 / 3}}$ & $\frac{1}{\left(w^{2}\right)^{4 / 3}}$ & $\frac{1}{\left(w^{2}\right)^{1 / 3}}$ & $\left(w^{2}\right)^{2 / 3}$ & $\left(w^{2}\right)^{5 / 3}$ & $\left(w^{2}\right)^{8 / 3}$ \\
\hline$w^{1}$ & $\frac{1}{w^{3}\left(w^{2}\right)^{1 / 3}}$ & $\frac{1}{w\left(w^{2}\right)^{1 / 3}}$ & $\frac{w}{\left(w^{2}\right)^{1 / 3}}$ & $w\left(w^{2}\right)^{2 / 3}$ & $w^{3}\left(w^{2}\right)^{2 / 3}$ & $w^{5}\left(w^{2}\right)^{2 / 3}$ \\
\hline$w^{2}$ & $\frac{1}{\left(w^{2}\right)^{4 / 3}}$ & $\frac{1}{\left(w^{2}\right)^{1 / 3}}$ & $\left(w^{2}\right)^{2 / 3}$ & $\left(w^{2}\right)^{5 / 3}$ & $\left(w^{2}\right)^{8 / 3}$ & $\left(w^{2}\right)^{11 / 3}$ \\
\hline$w^{3}$ & $\frac{1}{w\left(w^{2}\right)^{1 / 3}}$ & $\frac{w}{\left(w^{2}\right)^{1 / 3}}$ & $w\left(w^{2}\right)^{2 / 3}$ & $w^{3}\left(w^{2}\right)^{2 / 3}$ & $w^{5}\left(w^{2}\right)^{2 / 3}$ & $w^{7}\left(w^{2}\right)^{2 / 3}$ \\
\hline
\end{tabular}


Table 2: Mathematica 8 default simplification for 1 st row $\times 1$ st column, with flaw numbers. Compare with Table 1 .

\begin{tabular}{|c|c|c|c|c|c|c|c|c|c|c|c|c|}
\hline $\overrightarrow{\downarrow x}$ & $\left(w^{2}\right)^{-7 / 3}$ & & $\left(w^{2}\right)^{-4 / 3}$ & & $\left(w^{2}\right)^{-1 / 3}$ & & $\left(w^{2}\right)^{2 / 3}$ & & $\left(w^{2}\right)^{5 / 3}$ & & $\left(w^{2}\right)^{8 / 3}$ & \\
\hline$w^{-3}$ & $\frac{1}{w^{3}\left(w^{2}\right)^{7 / 3}}$ & $\begin{array}{l}2 \\
5 \\
6\end{array}$ & $\frac{1}{w^{3}\left(w^{2}\right)^{4 / 3}}$ & $\begin{array}{l}2 \\
5 \\
6 \\
\end{array}$ & $\frac{1}{w^{3}\left(w^{2}\right)^{1 / 3}}$ & 2 & $\frac{\left(w^{2}\right)^{2 / 3}}{w^{3}}$ & \begin{tabular}{|l} 
\\
3 \\
5 \\
\end{tabular} & $\frac{\left(w^{2}\right)^{5 / 3}}{w^{3}}$ & $\begin{array}{l}2 \\
3 \\
5\end{array}$ & $\frac{\left(w^{2}\right)^{8 / 3}}{w^{3}}$ & $\begin{array}{l}2 \\
3 \\
5 \\
6 \\
\end{array}$ \\
\hline$w^{-2}$ & $\frac{1}{w^{2}\left(w^{2}\right)^{7 / 3}}$ & \begin{tabular}{|l}
2 \\
4 \\
5 \\
6 \\
7 \\
\end{tabular} & $\frac{1}{w^{2}\left(w^{2}\right)^{4 / 3}}$ & \begin{tabular}{|l|}
$\mathbf{2}$ \\
4 \\
5 \\
6 \\
7 \\
\end{tabular} & $\frac{1}{w^{2}\left(w^{2}\right)^{1 / 3}}$ & $\begin{array}{l}2 \\
4 \\
6 \\
7\end{array}$ & $\frac{\left(w^{2}\right)^{2 / 3}}{w^{2}}$ & \begin{tabular}{|l}
2 \\
3 \\
4 \\
5 \\
6 \\
\end{tabular} & $\frac{\left(w^{2}\right)^{5 / 3}}{w^{2}}$ & $\begin{array}{l}\overline{2} \\
3 \\
4 \\
5\end{array}$ & $\frac{\left(w^{2}\right)^{8 / 3}}{w^{2}}$ & \begin{tabular}{|l|}
2 \\
3 \\
4 \\
5 \\
6 \\
\end{tabular} \\
\hline$w^{-1}$ & $\frac{1}{w\left(w^{2}\right)^{7 / 3}}$ & $\mid \begin{array}{l}2 \\
5 \\
6\end{array}$ & $\frac{1}{w\left(w^{2}\right)^{4 / 3}}$ & $\begin{array}{l}2 \\
5 \\
6\end{array}$ & $\frac{1}{w\left(w^{2}\right)^{1 / 3}}$ & 2 & $\frac{\left(w^{2}\right)^{2 / 3}}{w}$ & \begin{tabular}{|l}
2 \\
3 \\
5 \\
\end{tabular} & $\frac{\left(w^{2}\right)^{5 / 3}}{w}$ & $\begin{array}{l}2 \\
3 \\
5 \\
\end{array}$ & $\frac{\left(w^{2}\right)^{8 / 3}}{w}$ & \begin{tabular}{|l}
2 \\
3 \\
5 \\
6
\end{tabular} \\
\hline$w^{0}$ & $\frac{1}{\left(w^{2}\right)^{7 / 3}}$ & 2 & $\frac{1}{\left(w^{2}\right)^{4 / 3}}$ & 2 & $\frac{1}{\left(w^{2}\right)^{1 / 3}}$ & 2 & $\left(w^{2}\right)^{2 / 3}$ & 2 & $\left(w^{2}\right)^{5 / 3}$ & 2 & $\left(w^{2}\right)^{8 / 3}$ & 2 \\
\hline$w^{1}$ & $\frac{w}{\left(w^{2}\right)^{7 / 3}}$ & $\begin{array}{l}2 \\
3 \\
5 \\
\end{array}$ & $\frac{w}{\left(w^{2}\right)^{4 / 3}}$ & \begin{tabular}{|l|}
2 \\
3 \\
5 \\
6 \\
\end{tabular} & $\frac{w}{\left(w^{2}\right)^{1 / 3}}$ & 2 & $w\left(w^{2}\right)^{2 / 3}$ & 2 & $w\left(w^{2}\right)^{5 / 3}$ & $\begin{array}{l}2 \\
5 \\
6\end{array}$ & $w\left(w^{2}\right)^{8 / 3}$ & $\begin{array}{l}2 \\
5 \\
6\end{array}$ \\
\hline$w^{2}$ & $\frac{1}{\left(w^{2}\right)^{4 / 3}}$ & 2 & $\frac{1}{\left(w^{2}\right)^{1 / 3}}$ & 2 & $\left(w^{2}\right)^{2 / 3}$ & 2 & $\left(w^{2}\right)^{5 / 3}$ & 2 & $\left(w^{2}\right)^{8 / 3}$ & 2 & $\left(w^{2}\right)^{11 / 3}$ & 2 \\
\hline$w^{3}$ & $\frac{w^{3}}{\left(w^{2}\right)^{7 / 3}}$ & $\begin{array}{l}2 \\
3 \\
5 \\
6\end{array}$ & $\frac{w^{3}}{\left(w^{2}\right)^{4 / 3}}$ & $\begin{array}{l}\mathbf{2} \\
3 \\
5 \\
6\end{array}$ & $\frac{w^{3}}{\left(w^{2}\right)^{1 / 3}}$ & $\begin{array}{l}\mathbf{2} \\
3 \\
6\end{array}$ & $w^{3}\left(w^{2}\right)^{2 / 3}$ & 2 & $w^{3}\left(w^{2}\right)^{5 / 3}$ & $\begin{array}{l}2 \\
5 \\
6\end{array}$ & $w^{3}\left(w^{2}\right)^{8 / 3}$ & $\begin{array}{l}2 \\
5 \\
6\end{array}$ \\
\hline
\end{tabular}

Table 3: Mathematica 8 FullSimplify[...] for 1st row $\times 1$ st column, with flaw numbers. Compare with Table 1 .

\begin{tabular}{|c|c|c|c|c|c|c|c|c|c|c|c|c|}
\hline $\overrightarrow{\downarrow x}$ & $\left(w^{2}\right)^{-7}$ & & $\left(w^{2}\right)^{-4}$ & & $\left(w^{2}\right)^{-1 / 3}$ & & $\left(w^{2}\right)^{2 / 3}$ & & $\left(w^{2}\right)^{5 / 3}$ & & $\left(w^{2}\right)^{8 / 3}$ & \\
\hline$w^{-3}$ & $\frac{\left(w^{2}\right)^{2 / 3}}{w^{9}}$ & $\begin{array}{l}1 \\
3 \\
5\end{array}$ & $\frac{\left(w^{2}\right)^{2 / 3}}{w^{7}}$ & $\begin{array}{l}1 \\
3 \\
5\end{array}$ & $\frac{\left(w^{2}\right)^{2 / 3}}{w^{5}}$ & $\begin{array}{l}1 \\
3 \\
5\end{array}$ & $\frac{\left(w^{2}\right)^{2 / 3}}{w^{3}}$ & $\begin{array}{l}3 \\
5\end{array}$ & $\frac{w}{\left(w^{2}\right)^{1 / 3}}$ & & $w\left(w^{2}\right)^{2 / 3}$ & \\
\hline$w^{-2}$ & $\frac{\left(w^{2}\right)^{2 / 3}}{w^{8}}$ & $\begin{array}{l}1 \\
3 \\
4 \\
5 \\
6\end{array}$ & $\frac{\left(w^{2}\right)^{2 / 3}}{w^{6}}$ & $\begin{array}{l}1 \\
3 \\
4 \\
5 \\
6\end{array}$ & $\frac{1}{\left(w^{2}\right)^{4 / 3}}$ & & $\frac{1}{\left(w^{2}\right)^{1 / 3}}$ & & $\left(w^{2}\right)^{2 / 3}$ & & $\left(w^{2}\right)^{5 / 3}$ & \\
\hline$w^{-1}$ & $\frac{\left(w^{2}\right)^{2 / 3}}{w^{7}}$ & $\begin{array}{l}1 \\
3 \\
5\end{array}$ & $\frac{\left(w^{2}\right)^{2 / 3}}{w^{5}}$ & $\begin{array}{l}1 \\
3 \\
5 \\
6\end{array}$ & $\frac{\left(w^{2}\right)^{2 / 3}}{w^{3}}$ & $\begin{array}{l}1 \\
3 \\
5 \\
6\end{array}$ & $\frac{w}{\left(w^{2}\right)^{1 / 3}}$ & & $w\left(w^{2}\right)^{2 / 3}$ & & $w^{3}\left(w^{2}\right)^{2 / 3}$ & \\
\hline$w^{0}$ & $\frac{1}{\left(w^{2}\right)^{7 / 3}}$ & & $\frac{1}{\left(w^{2}\right)^{4 / 3}}$ & & $\frac{1}{\left(w^{2}\right)^{1 / 3}}$ & & $\left(w^{2}\right)^{2 / 3}$ & & $\left(w^{2}\right)^{5 / 3}$ & & $\left(w^{2}\right)^{8 / 3}$ & \\
\hline$w^{1}$ & $\frac{w}{\left(w^{2}\right)^{7 / 3}}$ & $\begin{array}{l}3 \\
5 \\
\end{array}$ & $\frac{w}{\left(w^{2}\right)^{4 / 3}}$ & $\begin{array}{l}3 \\
5\end{array}$ & $\frac{w}{\left(w^{2}\right)^{1 / 3}}$ & & $w\left(w^{2}\right)^{2 / 3}$ & & $w\left(w^{2}\right)^{5 / 3}$ & $\begin{array}{l}5 \\
6\end{array}$ & $w\left(w^{2}\right)^{8 / 3}$ & $\begin{array}{l}5 \\
6\end{array}$ \\
\hline$w^{2}$ & $\frac{1}{\left(w^{2}\right)^{4 / 3}}$ & & $\frac{1}{\left(w^{2}\right)^{1 / 3}}$ & & $\left(w^{2}\right)^{2 / 3}$ & & $\left(w^{2}\right)^{5 / 3}$ & & $\left(w^{2}\right)^{8 / 3}$ & & $\left(w^{2}\right)^{11 / 3}$ & \\
\hline$w^{3}$ & $\frac{w^{3}}{\left(w^{2}\right)^{7 / 3}}$ & $\begin{array}{l}3 \\
5 \\
6 \\
8\end{array}$ & $\frac{w}{\left(w^{2}\right)^{1 / 3}}$ & & $w\left(w^{2}\right)^{2 / 3}$ & & $w^{3}\left(w^{2}\right)^{2 / 3}$ & & $w^{3}\left(w^{2}\right)^{5 / 3}$ & $\begin{array}{l}5 \\
6\end{array}$ & $w^{3}\left(w^{2}\right)^{8 / 3}$ & $\begin{array}{l}5 \\
6\end{array}$ \\
\hline
\end{tabular}


Table 4: Derive 6 default simplify for 1 st row $\times 1$ st column, with flaw numbers.

Compare with Table 1 .

\begin{tabular}{|c|c|c|c|c|c|c|c|c|c|c|c|}
\hline $\overrightarrow{\downarrow x}$ & $\left(w^{2}\right)^{-7}$ & & $\left(w^{2}\right)^{-4}$ & & $\left(w^{2}\right)^{-1 /}$ & & $\left(w^{2}\right)^{2 / 3}$ & & $\left(w^{2}\right)^{5 / 3}$ & & $\left(w^{2}\right)^{8 / 3}$ \\
\hline$w^{-3}$ & $\frac{\left(w^{2}\right)^{2 / 3}}{w^{9}}$ & $\begin{array}{l}1 \\
3 \\
5\end{array}$ & $\frac{\left(w^{2}\right)^{2 / 3}}{w^{7}}$ & $\begin{array}{l}1 \\
3 \\
5\end{array}$ & $\frac{\left(w^{2}\right)^{2 / 3}}{w^{5}}$ & $\begin{array}{l}\mathbf{1} \\
3 \\
5\end{array}$ & $\frac{\left(w^{2}\right)^{2 / 3}}{w^{3}}$ & $\begin{array}{l}3 \\
5\end{array}$ & $\frac{\left(w^{2}\right)^{2 / 3}}{w}$ & $\begin{array}{l}3 \\
5\end{array}$ & $w\left(w^{2}\right)^{2 / 3}$ \\
\hline$w^{-2}$ & $\frac{1}{\left(w^{2}\right)^{10 / 3}}$ & & $\frac{1}{\left(w^{2}\right)^{7 / 3}}$ & & $\frac{1}{\left(w^{2}\right)^{4 / 3}}$ & & $\frac{1}{\left(w^{2}\right)^{1 / 3}}$ & & $\left(w^{2}\right)^{2 / 3}$ & & $\left(w^{2}\right)^{5 / 3}$ \\
\hline$w^{-1}$ & $\frac{\left(w^{2}\right)^{2 / 3}}{w^{7}}$ & $\begin{array}{l}\mathbf{1} \\
3 \\
5\end{array}$ & $\frac{\left(w^{2}\right)^{2 / 3}}{w^{5}}$ & $\begin{array}{l}1 \\
3 \\
5\end{array}$ & $\frac{\left(w^{2}\right)^{2 / 3}}{w^{3}}$ & $\begin{array}{l}1 \\
3 \\
5\end{array}$ & $\frac{\left(w^{2}\right)^{2 / 3}}{w}$ & $\begin{array}{l}3 \\
5\end{array}$ & $w\left(w^{2}\right)^{2 / 3}$ & & $w^{3}\left(w^{2}\right)^{2 / 3}$ \\
\hline$w^{0}$ & $\frac{1}{\left(w^{2}\right)^{7 / 3}}$ & & $\frac{1}{\left(w^{2}\right)^{4 / 3}}$ & & $\frac{1}{\left(w^{2}\right)^{1 / 3}}$ & & $\left(w^{2}\right)^{2 / 3}$ & & $\left(w^{2}\right)^{5 / 3}$ & & $\left(w^{2}\right)^{8 / 3}$ \\
\hline$w^{1}$ & $\frac{\left(w^{2}\right)^{2 / 3}}{w^{5}}$ & $\begin{array}{l}3 \\
5 \\
\end{array}$ & $\frac{\left(w^{2}\right)^{2 / 3}}{w^{3}}$ & $\begin{array}{l}3 \\
5 \\
\end{array}$ & $\frac{\left(w^{2}\right)^{2 / 3}}{w}$ & $\begin{array}{l}3 \\
5\end{array}$ & $w\left(w^{2}\right)^{2 / 3}$ & & $w^{3}\left(w^{2}\right)^{2 / 3}$ & & $w^{5}\left(w^{2}\right)^{2 / 3}$ \\
\hline$w^{2}$ & $\frac{1}{\left(w^{2}\right)^{4 / 3}}$ & & $\frac{1}{\left(w^{2}\right)^{1 / 3}}$ & & $\left(w^{2}\right)^{2 / 3}$ & & $\left(w^{2}\right)^{5 / 3}$ & & $\left(w^{2}\right)^{8 / 3}$ & & $\left(w^{2}\right)^{11 / 3}$ \\
\hline$w^{3}$ & $\frac{\left(w^{2}\right)^{2 / 3}}{w^{3}}$ & $\begin{array}{l}3 \\
5 \\
\end{array}$ & $\frac{\left(w^{2}\right)^{2 / 3}}{w}$ & $\begin{array}{l}3 \\
5 \\
\end{array}$ & $w\left(w^{2}\right)^{2 / 3}$ & & $w^{3}\left(w^{2}\right)^{2 / 3}$ & & $w^{5}\left(w^{2}\right)^{2 / 3}$ & & $w^{7}\left(w^{2}\right)^{2 / 3}$ \\
\hline
\end{tabular}

Table 5: TI-CAS 3.1 default simplify for 1 st row $\times 1$ st column, with flaw numbers. Compare with Table 1

\begin{tabular}{|c|c|c|c|c|c|c|c|c|c|}
\hline$\vec{\downarrow} \vec{x}$ & $\left(w^{2}\right)^{-7 / 3}$ & $\left(w^{2}\right)^{-4 / 5}$ & $\left(w^{2}\right)^{-1}$ & & $\left(w^{2}\right)^{2 / 3}$ & & $\left(w^{2}\right)^{5 / 3}$ & & $\left(w^{2}\right)^{8 / 3}$ \\
\hline$w^{-3}$ & $\frac{1}{w^{7}\left(w^{2}\right)^{1 / 3}}$ & $\frac{1}{w^{5}\left(w^{2}\right)^{1 / 3}}$ & $\frac{1}{w^{3}\left(w^{2}\right)^{1 / 3}}$ & 2 & $\frac{\left(w^{2}\right)^{2 / 3}}{w^{3}}$ & $\begin{array}{l}2 \\
3 \\
5 \\
6\end{array}$ & $\frac{\left(w^{2}\right)^{2 / 3}}{w}$ & $\begin{array}{l}3 \\
5 \\
6\end{array}$ & $w\left(w^{2}\right)^{2 / 3}$ \\
\hline$w^{-2}$ & $\frac{1}{\left(w^{2}\right)^{10 / 3}}$ & $\frac{1}{\left(w^{2}\right)^{7 / 3}}$ & $\frac{1}{\left(w^{2}\right)^{4 / 3}}$ & & $\frac{1}{\left(w^{2}\right)^{1 / 3}}$ & & $\left(w^{2}\right)^{2 / 3}$ & & $\left(w^{2}\right)^{5 / 3}$ \\
\hline$w^{-1}$ & $\frac{1}{w^{5}\left(w^{2}\right)^{1 / 3}}$ & $\frac{1}{w^{3}\left(w^{2}\right)^{1 / 3}}$ & $\frac{1}{w\left(w^{2}\right)^{1 / 3}}$ & 2 & $\frac{\left(w^{2}\right)^{2 / 3}}{w}$ & $\begin{array}{l}\mathbf{2} \\
3 \\
5 \\
6\end{array}$ & $w\left(w^{2}\right)^{2 / 3}$ & & $w^{3}\left(w^{2}\right)^{2 / 3}$ \\
\hline$w^{0}$ & $\frac{1}{\left(w^{2}\right)^{7 / 3}}$ & $\frac{1}{\left(w^{2}\right)^{4 / 3}}$ & $\frac{1}{\left(w^{2}\right)^{1 / 3}}$ & & $\left(w^{2}\right)^{2 / 3}$ & & $\left(w^{2}\right)^{5 / 3}$ & & $\left(w^{2}\right)^{8 / 3}$ \\
\hline$w^{1}$ & $\frac{1}{w^{3}\left(w^{2}\right)^{1 / 3}}$ & $\frac{1}{w\left(w^{2}\right)^{1 / 3}}$ & $\frac{w}{\left(w^{2}\right)^{1 / 3}}$ & 2 & $w\left(w^{2}\right)^{2 / 3}$ & 2 & $w^{3}\left(w^{2}\right)^{2 / 3}$ & & $w^{5}\left(w^{2}\right)^{2 / 3}$ \\
\hline$w^{2}$ & $\frac{1}{\left(w^{2}\right)^{4 / 3}}$ & $\frac{1}{\left(w^{2}\right)^{1 / 3}}$ & $\left(w^{2}\right)^{2 / 3}$ & & $\left(w^{2}\right)^{5 / 3}$ & & $\left(w^{2}\right)^{8 / 3}$ & & $\left(w^{2}\right)^{11 / 3}$ \\
\hline$w^{3}$ & $\frac{1}{w\left(w^{2}\right)^{1 / 3}}$ & $\frac{w}{\left(w^{2}\right)^{1 / 3}}$ & $\frac{w^{3}}{\left(w^{2}\right)^{1 / 3}}$ & $\begin{array}{l}2 \\
3 \\
6\end{array}$ & $w^{3}\left(w^{2}\right)^{2 / 3}$ & 2 & $w^{5}\left(w^{2}\right)^{2 / 3}$ & & $w^{7}\left(w^{2}\right)^{2 / 3}$ \\
\hline
\end{tabular}


Table 6: Maple 15 and Maxima 5.24 default simplification for 1 st row $\times 1$ st column, with flaw numbers. Compare with Table 1 .

\begin{tabular}{|c|c|c|c|c|c|c|c|c|c|c|c|c|}
\hline$\vec{x}$ & $\left(w^{2}\right)^{-7 / 3}$ & & $\left(w^{2}\right)^{-4 / i}$ & & $\left(w^{2}\right)^{-1 / 3}$ & & $\left(w^{2}\right)^{2 / 3}$ & & $\left(w^{2}\right)^{5 / 3}$ & & $\left(w^{2}\right)^{8 / 3}$ & \\
\hline$w^{-3}$ & $\frac{1}{w^{3}\left(w^{2}\right)^{7 / 3}}$ & $\begin{array}{l}2 \\
5 \\
6\end{array}$ & $\frac{1}{w^{3}\left(w^{2}\right)^{4 / 3}}$ & $\begin{array}{l}2 \\
5 \\
6\end{array}$ & $\frac{1}{w^{3}\left(w^{2}\right)^{1 / 3}}$ & 2 & $\frac{\left(w^{2}\right)^{2 / 3}}{w^{3}}$ & $\begin{array}{l}2 \\
3 \\
5 \\
6 \\
\end{array}$ & $\frac{\left(w^{2}\right)^{5 / 3}}{w^{3}}$ & \begin{tabular}{|l|}
2 \\
3 \\
5 \\
6 \\
\end{tabular} & $\frac{\left(w^{2}\right)^{8 / 3}}{w^{3}}$ & $\begin{array}{l}2 \\
3 \\
5 \\
6 \\
\end{array}$ \\
\hline$w^{-2}$ & $\frac{1}{w^{2}\left(w^{2}\right)^{7 / 3}}$ & $\begin{array}{l}2 \\
4 \\
5 \\
6\end{array}$ & $\frac{1}{w^{2}\left(w^{2}\right)^{4 / 3}}$ & $\begin{array}{l}2 \\
4 \\
5 \\
6\end{array}$ & $\frac{1}{w^{2}\left(w^{2}\right)^{1 / 3}}$ & $\begin{array}{l}2 \\
4 \\
6\end{array}$ & $\frac{\left(w^{2}\right)^{2 / 3}}{w^{2}}$ & $\begin{array}{l}2 \\
3 \\
4 \\
5 \\
6\end{array}$ & $\frac{\left(w^{2}\right)^{5 / 3}}{w^{2}}$ & \begin{tabular}{|l|}
2 \\
3 \\
4 \\
5 \\
6 \\
\end{tabular} & $\frac{\left(w^{2}\right)^{8 / 3}}{w^{2}}$ & $\begin{array}{l}2 \\
3 \\
4 \\
5 \\
6\end{array}$ \\
\hline$w^{-1}$ & $\frac{1}{w\left(w^{2}\right)^{7 / 3}}$ & $\begin{array}{l}2 \\
5 \\
6\end{array}$ & $\frac{1}{w\left(w^{2}\right)^{4 / 3}}$ & $\mid \begin{array}{l}2 \\
5 \\
6\end{array}$ & $\frac{1}{w\left(w^{2}\right)^{1 / 3}}$ & 2 & $\frac{\left(w^{2}\right)^{2 / 3}}{w}$ & $\begin{array}{l}2 \\
3 \\
5 \\
6\end{array}$ & $\frac{\left(w^{2}\right)^{5 / 3}}{w}$ & $\begin{array}{l}2 \\
3 \\
5 \\
6 \\
\end{array}$ & $\frac{\left(w^{2}\right)^{8 / 3}}{w}$ & $\begin{array}{l}2 \\
3 \\
5 \\
6\end{array}$ \\
\hline$w^{0}$ & $\frac{1}{\left(w^{2}\right)^{7 / 3}}$ & 2 & $\frac{1}{\left(w^{2}\right)^{4 / 3}}$ & 2 & $\frac{1}{\left(w^{2}\right)^{1 / 3}}$ & 2 & $\left(w^{2}\right)^{2 / 3}$ & 2 & $\left(w^{2}\right)^{5 / 3}$ & 2 & $\left(w^{2}\right)^{8 / 3}$ & 2 \\
\hline$w^{1}$ & $\frac{w}{\left(w^{2}\right)^{7 / 3}}$ & $\begin{array}{l}2 \\
3 \\
5 \\
6\end{array}$ & $\frac{w}{\left(w^{2}\right)^{4 / 3}}$ & $\begin{array}{l}2 \\
3 \\
5 \\
6\end{array}$ & $\frac{w}{\left(w^{2}\right)^{1 / 3}}$ & 2 & $w\left(w^{2}\right)^{2 / 3}$ & 2 & $w\left(w^{2}\right)^{5 / 3}$ & $\begin{array}{l}2 \\
5 \\
6\end{array}$ & $w\left(w^{2}\right)^{8 / 3}$ & $\begin{array}{l}2 \\
5 \\
6\end{array}$ \\
\hline$w^{2}$ & $\frac{w^{2}}{\left(w^{2}\right)^{7 / 3}}$ & \begin{tabular}{|l}
$\mathbf{2}$ \\
3 \\
4 \\
5 \\
6 \\
8 \\
\end{tabular} & $\frac{w^{2}}{\left(w^{2}\right)^{4 / 3}}$ & $\begin{array}{l}\mathbf{2} \\
3 \\
4 \\
5 \\
6 \\
8 \\
\end{array}$ & $\frac{w^{2}}{\left(w^{2}\right)^{1 / 3}}$ & $\begin{array}{l}2 \\
3 \\
4 \\
6\end{array}$ & $w^{2}\left(w^{2}\right)^{2 / 3}$ & $\begin{array}{l}2 \\
4 \\
6\end{array}$ & $w^{2}\left(w^{2}\right)^{5 / 3}$ & $\mid \begin{array}{l}2 \\
4 \\
5 \\
6\end{array}$ & $w^{2}\left(w^{2}\right)^{8 / 3}$ & $\begin{array}{l}2 \\
4 \\
5 \\
6\end{array}$ \\
\hline$w^{3}$ & $\frac{w^{3}}{\left(w^{2}\right)^{7 / 3}}$ & $\begin{array}{l}2 \\
3 \\
5 \\
6\end{array}$ & $\frac{w^{3}}{\left(w^{2}\right)^{4 / 3}}$ & \begin{tabular}{|l}
2 \\
3 \\
5 \\
6
\end{tabular} & $\frac{w^{3}}{\left(w^{2}\right)^{1 / 3}}$ & $\begin{array}{l}2 \\
3 \\
6\end{array}$ & $w^{3}\left(w^{2}\right)^{2 / 3}$ & 2 & $w^{3}\left(w^{2}\right)^{5 / 3}$ & $\begin{array}{l}2 \\
5\end{array}$ & $w^{3}\left(w^{2}\right)^{8 / 3}$ & $\begin{array}{l}2 \\
5 \\
6\end{array}$ \\
\hline
\end{tabular}

Table 7: Maxima 5.24 fullratsimp(...) and Maple 15 simplify(...) for 1st row $\times$ 1st column, with flaw numbers. Compare with Table 1.

\begin{tabular}{|c|c|c|c|c|c|c|c|c|c|c|c|c|}
\hline $\overrightarrow{\downarrow x}$ & $\left(w^{2}\right)^{-7 / 3}$ & & $\left(w^{2}\right)^{-4 / 3}$ & & $\left(w^{2}\right)^{-1 / 3}$ & & $\left(w^{2}\right)^{2 / 3}$ & & $\left(w^{2}\right)^{5 / 3}$ & & $\left(w^{2}\right)^{8 / 3}$ & \\
\hline$w^{-3}$ & $\frac{1}{w^{7}\left(w^{2}\right)^{1 / 3}}$ & & $\frac{1}{w^{5}\left(w^{2}\right)^{1 / 3}}$ & & $\frac{1}{w^{3}\left(w^{2}\right)^{1 / 3}}$ & & $\frac{\left(w^{2}\right)^{2 / 3}}{w^{3}}$ & $\begin{array}{l}3 \\
5 \\
6\end{array}$ & $\frac{\left(w^{2}\right)^{2 / 3}}{w}$ & $\begin{array}{l}3 \\
5 \\
6\end{array}$ & $w\left(w^{2}\right)^{2 / 3}$ & \\
\hline$w^{-2}$ & $\frac{1}{w^{6}\left(w^{2}\right)^{1 / 3}}$ & 4 & $\frac{1}{w^{4}\left(w^{2}\right)^{1 / 3}}$ & 4 & $\frac{1}{w^{2}\left(w^{2}\right)^{1 / 3}}$ & 4 & $\frac{\left(w^{2}\right)^{2 / 3}}{w^{2}}$ & \begin{tabular}{|l|}
3 \\
4 \\
5 \\
\end{tabular} & $\left(w^{2}\right)^{2 / 3}$ & & $w^{2}\left(w^{2}\right)^{2 / 3}$ & 4 \\
\hline$w^{-1}$ & $\frac{1}{w^{5}\left(w^{2}\right)^{1 / 3}}$ & & $\frac{1}{w^{3}\left(w^{2}\right)^{1 / 3}}$ & & $\frac{1}{w\left(w^{2}\right)^{1 / 3}}$ & & $\frac{\left(w^{2}\right)^{2 / 3}}{w}$ & $\begin{array}{l}3 \\
5 \\
6\end{array}$ & $w\left(w^{2}\right)^{2 / 3}$ & & $w^{3}\left(w^{2}\right)^{2 / 3}$ & \\
\hline$w^{0}$ & $\frac{1}{w^{4}\left(w^{2}\right)^{1 / 3}}$ & 4 & $\frac{1}{w^{2}\left(w^{2}\right)^{1 / 3}}$ & & $\frac{1}{\left(w^{2}\right)^{1 / 3}}$ & & $\left(w^{2}\right)^{2 / 3}$ & & $w^{2}\left(w^{2}\right)^{2 / 3}$ & 4 & $w^{4}\left(w^{2}\right)^{2 / 3}$ & 4 \\
\hline$w^{1}$ & $\frac{1}{w^{3}\left(w^{2}\right)^{1 / 3}}$ & & $\frac{1}{w\left(w^{2}\right)^{1 / 3}}$ & & $\frac{w}{\left(w^{2}\right)^{1 / 3}}$ & & $w\left(w^{2}\right)^{2 / 3}$ & & $w^{3}\left(w^{2}\right)^{2 / 3}$ & & $w^{5}\left(w^{2}\right)^{2 / 3}$ & \\
\hline$w^{2}$ & $\frac{1}{w^{2}\left(w^{2}\right)^{1 / 3}}$ & 4 & $\frac{1}{\left(w^{2}\right)^{1 / 3}}$ & & $\frac{w^{2}}{\left(w^{2}\right)^{1 / 3}}$ & 4 & $w^{2}\left(w^{2}\right)^{2 / 3}$ & 4 & $w^{4}\left(w^{2}\right)^{2 / 3}$ & 4 & $w^{6}\left(w^{2}\right)^{2 / 3}$ & 4 \\
\hline$w^{3}$ & $\frac{1}{w\left(w^{2}\right)^{1 / 3}}$ & & $\frac{w}{\left(w^{2}\right)^{1 / 3}}$ & & $\frac{w^{3}}{\left(w^{2}\right)^{1 / 3}}$ & & $w^{3}\left(w^{2}\right)^{2 / 3}$ & & $w^{5}\left(w^{2}\right)^{2 / 3}$ & & $w^{7}\left(w^{2}\right)^{2 / 3}$ & \\
\hline
\end{tabular}


Table 8: Unflawed results of Appendix rewrite rules for 1 st row $\times 1$ st column. Compare with Tables 9 through 15.

\begin{tabular}{|c||c|c|c|c|c|c|}
\hline$\vec{\downarrow}$ & $\left(w^{-2}\right)^{-5 / 2}$ & $\left(w^{-2}\right)^{-3 / 2}$ & $\left(w^{-2}\right)^{-1 / 2}$ & $\left(w^{-2}\right)^{1 / 2}$ & $\left(w^{-2}\right)^{3 / 2}$ & $\left(w^{-2}\right)^{5 / 2}$ \\
\hline \hline$w^{-3}$ & $\frac{w}{\sqrt{\frac{1}{w^{2}}}}$ & $\sqrt{\frac{1}{w^{2}}} w$ & $\frac{\sqrt{\frac{1}{w^{2}}}}{w}$ & $\frac{\sqrt{\frac{1}{w^{2}}}}{w^{3}}$ & $\frac{\sqrt{\frac{1}{w^{2}}}}{w^{5}}$ & $\frac{\sqrt{\frac{1}{w^{2}}}}{w^{7}}$ \\
\hline$w^{-2}$ & $\frac{1}{\left(\frac{1}{w^{2}}\right)^{3 / 2}}$ & $\frac{1}{\sqrt{\frac{1}{w^{2}}}}$ & $\sqrt{\frac{1}{w^{2}}}$ & $\left(\frac{1}{w^{2}}\right)^{3 / 2}$ & $\left(\frac{1}{w^{2}}\right)^{5 / 2}$ & $\left(\frac{1}{w^{2}}\right)^{7 / 2}$ \\
\hline$w^{-1}$ & $\frac{w^{3}}{\sqrt{\frac{1}{w^{2}}}}$ & $\frac{w}{\sqrt{\frac{1}{w^{2}}}}$ & $\sqrt{\frac{1}{w^{2}}} w$ & $\frac{\sqrt{\frac{1}{w^{2}}}}{w}$ & $\frac{\sqrt{\frac{1}{w^{2}}}}{w^{3}}$ & $\frac{\sqrt{\frac{1}{w^{2}}}}{w^{5}}$ \\
\hline$w^{0}$ & $\frac{1}{\left(\frac{1}{w^{2}}\right)^{5 / 2}}$ & $\frac{1}{\left(\frac{1}{w^{2}}\right)^{3 / 2}}$ & $\frac{1}{\sqrt{\frac{1}{w^{2}}}}$ & $\sqrt{\frac{1}{w^{2}}}$ & $\left(\frac{1}{w^{2}}\right)^{3 / 2}$ & $\left(\frac{1}{w^{2}}\right)^{5 / 2}$ \\
\hline$w^{1}$ & $\frac{w^{5}}{\sqrt{\frac{1}{w^{2}}}}$ & $\frac{w^{3}}{\sqrt{\frac{1}{w^{2}}}}$ & $\frac{w}{\sqrt{\frac{1}{w^{2}}}}$ & $\sqrt{\frac{1}{w^{2}}} w$ & $\frac{\sqrt{\frac{1}{w^{2}}}}{w}$ & $\frac{\sqrt{\frac{1}{w^{2}}}}{w^{3}}$ \\
\hline$w^{2}$ & $\frac{1}{\left(\frac{1}{w^{2}}\right)^{7 / 2}}$ & $\frac{1}{\left(\frac{1}{w^{2}}\right)^{5 / 2}}$ & $\frac{1}{\left(\frac{1}{w^{2}}\right)^{3 / 2}}$ & $\frac{1}{\sqrt{\frac{1}{w^{2}}}}$ & $\sqrt{\frac{1}{w^{2}}}$ & $\left(\frac{1}{w^{2}}\right)^{3 / 2}$ \\
\hline$w^{3}$ & $\frac{w^{7}}{\sqrt{\frac{1}{w^{2}}}}$ & $\frac{w^{5}}{\sqrt{\frac{1}{w^{2}}}}$ & $\frac{w^{3}}{\sqrt{\frac{1}{w^{2}}}}$ & $\frac{w}{\sqrt{\frac{1}{w^{2}}}}$ & $\sqrt{\frac{1}{w^{2}}} w$ & $\frac{\sqrt{\frac{1}{w^{2}}}}{w}$ \\
\hline
\end{tabular}

Table 9: Mathematica 8 default simplify for 1 st row $\times 1$ st column, with flaw numbers. Compare with Table 8 .

\begin{tabular}{|c|c|c|c|c|c|c|c|c|c|c|}
\hline$\sqrt{\vec{x}}$ & $\left(w^{-2}\right)^{-5 /}$ & & $\left(w^{-2}\right)^{-3 / 2}$ & & $\left(w^{-2}\right)^{-}$ & & $\left(w^{-2}\right)^{1 / 2}$ & $\left(w^{-2}\right)^{3 / 2}$ & & $\left(w^{-2}\right)^{5 / 2}$ \\
\hline$w^{-3}$ & $\frac{1}{\left(\frac{1}{w^{2}}\right)^{5 / 2} w^{3}}$ & $\left|\begin{array}{l}2 \\
3 \\
5 \\
6 \\
8\end{array}\right|$ & $\frac{1}{\left(\frac{1}{w^{2}}\right)^{3 / 2} w^{3}}$ & $\mid \begin{array}{l}2 \\
3 \\
5 \\
6 \\
8 \\
8 \\
1 \\
1\end{array}$ & $\frac{1}{\sqrt{\frac{1}{w^{2}}} w^{3}}$ & $\begin{array}{l}2 \\
3 \\
6 \\
8\end{array}$ & $\frac{\sqrt{\frac{1}{w^{2}}}}{w^{3}}$ & $\frac{\left(\frac{1}{w^{2}}\right)^{3 / 2}}{w^{3}}$ & $\begin{array}{l}2 \\
5 \\
6\end{array}$ & $\frac{\left(\frac{1}{w^{2}}\right)^{5 / 2}}{w^{3}}$ \\
\hline$w^{-2}$ & $\frac{1}{\left(\frac{1}{w^{2}}\right)^{3 / 2}}$ & 2 & $\frac{1}{\sqrt{\frac{1}{w^{2}}}}$ & 2 & $\sqrt{\frac{1}{w^{2}}}$ & 2 & $\left(\frac{1}{w^{2}}\right)^{3 / 2}$ & $\left(\frac{1}{w^{2}}\right)^{5 / 2}$ & 2 & $\left(\frac{1}{w^{2}}\right)^{7 / 2}$ \\
\hline$w^{-1}$ & $\frac{1}{\left(\frac{1}{w^{2}}\right)^{5 / 2} w}$ & $\mid \begin{array}{l}2 \\
3 \\
5 \\
6 \\
8 \\
8 \\
1 \\
1\end{array}$ & $\frac{1}{\left(\frac{1}{w^{2}}\right)^{3 / 2} w}$ & $\begin{array}{l}2 \\
\beta \\
\beta\end{array}$ & $\frac{1}{\sqrt{\frac{1}{w^{2}}} w}$ & $\begin{array}{l}2 \\
6 \\
8\end{array}$ & $\frac{\sqrt{\frac{1}{w^{2}}}}{w}$ & $\frac{\left(\frac{1}{w^{2}}\right)^{3 / 2}}{w}$ & $\begin{array}{l}2 \\
5 \\
6\end{array}$ & $\frac{\left(\frac{1}{w^{2}}\right)^{5 / 2}}{w}$ \\
\hline$w^{0}$ & $\frac{1}{\left(\frac{1}{w^{2}}\right)^{5 / 2}}$ & 2 & $\frac{1}{\left(\frac{1}{w^{2}}\right)^{3 / 2}}$ & 2 & $\frac{1}{\sqrt{\frac{1}{w^{2}}}}$ & 2 & $\sqrt{\frac{1}{w^{2}}}$ & $\left(\frac{1}{w^{2}}\right)^{3 / 2}$ & 2 & $\left(\frac{1}{w^{2}}\right)^{5 / 2}$ \\
\hline$w^{1}$ & $\frac{w}{\left(\frac{1}{w^{2}}\right)^{5 / 2}}$ & $\begin{array}{l}2 \\
5 \\
6 \\
\end{array}$ & $\frac{w}{\left(\frac{1}{w^{2}}\right)^{3 / 2}}$ & $\left|\begin{array}{l}2 \\
5 \\
6\end{array}\right|$ & $\frac{w}{\sqrt{\frac{1}{w^{2}}}}$ & 2 & $\sqrt{\frac{1}{w^{2}}} w$ & $\left(\frac{1}{w^{2}}\right)^{3 / 2} w$ & ${ }_{3}^{2}$ & $\left(\frac{1}{w^{2}}\right)^{5 / 2} w$ \\
\hline$w^{2}$ & $\frac{w^{2}}{\left(\frac{1}{w^{2}}\right)^{5 / 2}}$ & $\mid \begin{array}{l}2 \\
4 \\
5 \\
6\end{array}$ & $\frac{w^{2}}{\left(\frac{1}{w^{2}}\right)^{3 / 2}}$ & {$\left[\begin{array}{l}2 \\
4 \\
5\end{array}\right]$} & $\frac{w^{2}}{\sqrt{\frac{1}{w^{2}}}}$ & $\begin{array}{l}2 \\
4 \\
6\end{array}$ & $\sqrt{\frac{1}{w^{2}}} w^{2}$ & $\left(\frac{1}{w^{2}}\right)^{3 / 2} w^{2}$ & $\begin{array}{l}3 \\
4 \\
\end{array}$ & $\left(\frac{1}{w^{2}}\right)^{5 / 2} w^{2}$ \\
\hline$w^{3}$ & $\frac{w^{3}}{\left(\frac{1}{w^{2}}\right)^{5 / 2}}$ & $\left|\begin{array}{l}2 \\
5 \\
6\end{array}\right|$ & $\frac{w^{3}}{\left(\frac{1}{w^{2}}\right)^{3 / 2}}$ & $\begin{array}{l}2 \\
5\end{array}$ & $\frac{w^{3}}{\sqrt{\frac{1}{w^{2}}}}$ & 2 & $\sqrt{\frac{1}{w^{2}}} w^{3}$ & \begin{tabular}{l|l}
2 & $\left(\frac{1}{w^{2}}\right)^{3 / 2} w^{3}$ \\
6
\end{tabular} & & $\left(\frac{1}{w^{2}}\right)^{5 / 2} w^{3}$ \\
\hline
\end{tabular}


Table 10: Mathematica 8 FullSimplify[...] for 1 st row $\times 1$ st column, with flaw numbers. Compare with Table 8 .

\begin{tabular}{|c|c|c|c|c|c|c|c|c|c|c|}
\hline $\overrightarrow{\downarrow \vec{x}}$ & $\left(w^{-2}\right)^{-5 /}$ & & $\left(w^{-2}\right)^{-3 / 2}$ & & $\left(w^{-2}\right)^{-1 / 2}$ & $\left(w^{-2}\right)^{1 / 2}$ & & $\left(w^{-2}\right)^{3 / 2}$ & & $\left(w^{-2}\right)^{5 / 2}$ \\
\hline$w^{-3}$ & $\sqrt{\frac{1}{w^{2}}} w^{3}$ & $\begin{array}{l}3 \\
6\end{array}$ & $\overline{\mid l \sqrt{\frac{1}{w^{2}}}} w$ & & $\mid \frac{\sqrt{\frac{1}{w^{2}}}}{w}$ & \begin{tabular}{|l|}
$\frac{\sqrt{\frac{1}{w^{2}}}}{w^{3}}$ \\
\end{tabular} & & $\frac{\left(\frac{1}{w^{2}}\right)^{3 / 2}}{w^{3}}$ & 6 & $\begin{array}{l}\frac{\left(\frac{1}{w^{2}}\right)^{5 / 2}}{w^{3}} \\
\end{array}$ \\
\hline$w^{-2}$ & $\frac{1}{\left(\frac{1}{w^{2}}\right)^{3 / 2}}$ & & $\frac{1}{\sqrt{\frac{1}{w^{2}}}}$ & & $\sqrt{\frac{1}{w^{2}}}$ & $\left(\frac{1}{w^{2}}\right)^{3 / 2}$ & & $\left(\frac{1}{w^{2}}\right)^{5 / 2}$ & & $\left(\frac{1}{w^{2}}\right)^{7 / 2}$ \\
\hline$w^{-1}$ & $\sqrt{\frac{1}{w^{2}}} w^{5}$ & $\begin{array}{l}3 \\
6 \\
6\end{array}$ & $\sqrt{\frac{1}{w^{2}}} w^{3}$ & $\begin{array}{l}5 \\
6 \\
6\end{array}$ & $\sqrt{\frac{1}{w^{2}}} w$ & $\frac{\sqrt{\frac{1}{w^{2}}}}{w}$ & & $\frac{\left(\frac{1}{w^{2}}\right)^{3 / 2}}{w}$ & & $\frac{\left(\frac{1}{w^{2}}\right)^{5 / 2}}{w}$ \\
\hline$w^{0}$ & $\frac{1}{\left(\frac{1}{w^{2}}\right)^{5 / 2}}$ & & $\frac{1}{\left(\frac{1}{w^{2}}\right)^{3 / 2}}$ & & $\frac{1}{\sqrt{\frac{1}{w^{2}}}}$ & $\sqrt{\frac{1}{w^{2}}}$ & & $\left(\frac{1}{w^{2}}\right)^{3 / 2}$ & & $\left(\frac{1}{w^{2}}\right)^{5 / 2}$ \\
\hline$w^{1}$ & $\frac{w}{\left(\frac{1}{w^{2}}\right)^{5 / 2}}$ & $\begin{array}{l}5 \\
6\end{array}$ & $\frac{w}{\left(\frac{1}{w^{2}}\right)^{3 / 2}}$ & $\begin{array}{l}5 \\
6 \\
\end{array}$ & $\frac{w}{\sqrt{\frac{1}{w^{2}}}}$ & $\sqrt{\frac{1}{w^{2}}} w$ & & $\left(\frac{1}{w^{2}}\right)^{3 / 2} w$ & & $\left(\frac{1}{w^{2}}\right)^{5 / 2} w$ \\
\hline$w^{2}$ & $\sqrt{\frac{1}{w^{2}}} w^{8}$ & 1 & $\sqrt{\frac{1}{w^{2}}} w^{6}$ & $\begin{array}{l}1 \\
3 \\
4 \\
4\end{array}$ & $\sqrt{\frac{1}{w^{2}}} w^{4}$ & $\sqrt{\frac{1}{w^{2}}} w^{2}$ & & $\sqrt{\frac{1}{w^{2}}}$ & & $\left(\frac{1}{w^{2}}\right)^{3 / 2}$ \\
\hline$w^{3}$ & $\sqrt{\frac{1}{w^{2}}} w^{9}$ & & $\sqrt{\frac{1}{w^{2}}} w^{7}$ & ${ }_{3}^{1}$ & $\sqrt{\frac{1}{w^{2}}} w^{5}$ & $\sqrt{\frac{1}{w^{2}}} w^{3}$ & & $\sqrt{\frac{1}{w^{2}}} w$ & & $\left(\frac{1}{w^{2}}\right)^{5 / 2} w^{3}$ \\
\hline
\end{tabular}

Table 11: Derive 6 default simplify for 1 st row $\times 1$ st column, with flaw numbers. Compare with Table 8 .

\begin{tabular}{|c|c|c|c|c|c|c|c|c|}
\hline$\sqrt{\vec{x}}$ & $\left(w^{-2}\right)^{-5 /}$ & & $\left(w^{-2}\right)^{-3 /}$ & & $\left(w^{-2}\right)^{-1 / 2}$ & $\left(w^{-2}\right)^{1 / 2}$ & $\left(w^{-2}\right)^{3 / 2}$ & $\left(w^{-2}\right)^{5 / 2}$ \\
\hline$w^{-3}$ & $w^{3} \sqrt{\frac{1}{w^{2}}}$ & 3 & $\sqrt{\frac{1}{w^{2}}} w$ & & $\frac{\sqrt{\frac{1}{w^{2}}}}{w}$ & $\frac{\sqrt{\frac{1}{w^{2}}}}{w^{3}}$ & $\frac{\sqrt{\frac{1}{w^{2}}}}{w^{5}}$ & $\frac{\sqrt{\frac{1}{w^{2}}}}{w^{7}}$ \\
\hline$w^{-2}$ & $\frac{1}{\left(\frac{1}{w^{2}}\right)^{3 / 2}}$ & & $\frac{1}{\sqrt{\frac{1}{w^{2}}}}$ & & $\sqrt{\frac{1}{w^{2}}}$ & $\left(\frac{1}{w^{2}}\right)^{3 / 2}$ & $\left(\frac{1}{w^{2}}\right)^{5 / 2}$ & $\left(\frac{1}{w^{2}}\right)^{7 / 2}$ \\
\hline$w^{-1}$ & $w^{5} \sqrt{\frac{1}{w^{2}}}$ & 3 & $w^{3} \sqrt{\frac{1}{w^{2}}}$ & 3 & $\sqrt{\frac{1}{w^{2}}} w$ & $\frac{\sqrt{\frac{1}{w^{2}}}}{w}$ & $\frac{\sqrt{\frac{1}{w^{2}}}}{w^{3}}$ & $\frac{\sqrt{\frac{1}{w^{2}}}}{w^{5}}$ \\
\hline$w^{0}$ & $\frac{1}{\left(\frac{1}{w^{2}}\right)^{5 / 2}}$ & & $\frac{1}{\left(\frac{1}{w^{2}}\right)^{3 / 2}}$ & & & $\sqrt{\frac{1}{w^{2}}}$ & $\left(\frac{1}{w^{2}}\right)^{3 / 2}$ & $\left(\frac{1}{w^{2}}\right)^{5 / 2}$ \\
\hline$w^{1}$ & $w^{7} \sqrt{\frac{1}{w^{2}}}$ & 3 & $w^{5} \sqrt{\frac{1}{w^{2}}}$ & 3 & $w^{3} \sqrt{\frac{1}{w^{2}}}$ & $\sqrt{\frac{1}{w^{2}}} w$ & $\frac{\sqrt{\frac{1}{w^{2}}}}{w}$ & $\frac{\sqrt{\frac{1}{w^{2}}}}{w^{3}}$ \\
\hline$w^{2}$ & $\frac{1}{\left(\frac{1}{w^{2}}\right)^{7 / 2}}$ & & $\frac{1}{\left(\frac{1}{w^{2}}\right)^{5 / 2}}$ & & $\frac{1}{\left(\frac{1}{w^{2}}\right)^{3 / 2}}$ & $\frac{1}{\sqrt{\frac{1}{w^{2}}}}$ & $\sqrt{\frac{1}{w^{2}}}$ & $\left(\frac{1}{w^{2}}\right)^{3 / 2}$ \\
\hline$w^{3}$ & $w^{9} \sqrt{\frac{1}{w^{2}}}$ & 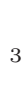 & $w^{7} \sqrt{\frac{1}{w^{2}}}$ & & $w^{5} \sqrt{\frac{1}{w^{2}}}$ & $w^{3} \sqrt{\frac{1}{w^{2}}}$ & $\sqrt{\frac{1}{w^{2}}} w$ & $\frac{\sqrt{\frac{1}{w^{2}}}}{w}$ \\
\hline
\end{tabular}


Table 12: TI-CAS and Maple default simplification for 1 st row $\times 1$ st column, with flaw numbers. Compare with Table 8 ,

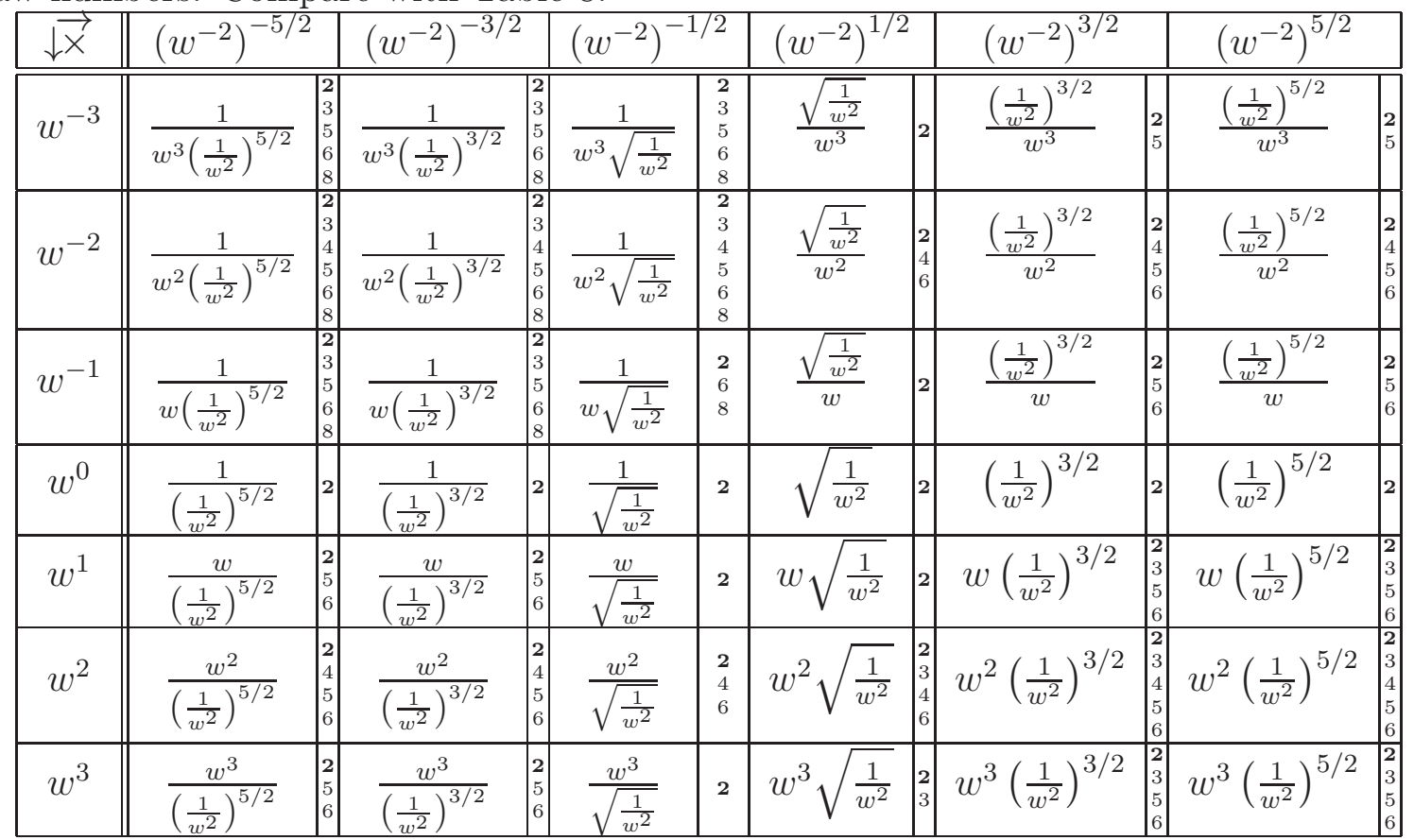

Table 13: Maxima $\mathbf{5 . 2 4}$ default simplify for 1 st row $\times$ 1st column, with flaw numbers. Compare with Table 8.

\begin{tabular}{|c|c|c|c|c|c|c|c|c|c|c|c|c|}
\hline $\overrightarrow{\downarrow x}$ & \multicolumn{2}{|l|}{$\left(w^{-2}\right)^{-5 / 2}$} & \multicolumn{2}{|l|}{$\left(w^{-2}\right)^{-3 / 2}$} & \multicolumn{2}{|c|}{$\left(w^{-2}\right)^{-1 / 2}$} & \multicolumn{2}{|c|}{$\left(w^{-2}\right)^{1 / 2}$} & \multicolumn{2}{|l|}{$\left(w^{-2}\right)^{3 / 2}$} & \multicolumn{2}{|c|}{$\left(w^{-2}\right)^{5 / 2}$} \\
\hline$w^{-3}$ & $\frac{\left(w^{2}\right)^{5 / 2}}{w^{3}}$ & \begin{tabular}{|l|}
$\mathbf{1}$ \\
$\mathbf{2}$ \\
3 \\
5 \\
6 \\
6
\end{tabular} & $\frac{\left(w^{2}\right)^{3 / 2}}{w^{3}}$ & \begin{tabular}{l|}
$\mathbf{1}$ \\
$\mathbf{2}$ \\
3 \\
5 \\
6 \\
\end{tabular} & $\frac{1}{\sqrt{\frac{1}{w^{2}}} w^{3}}$ & $\begin{array}{l}2 \\
3 \\
6 \\
8\end{array}$ & $\frac{\sqrt{\frac{1}{w^{2}}}}{w^{3}}$ & 2 & $\frac{1}{w^{3}\left(w^{2}\right)^{3 / 2}}$ & $\begin{array}{l}\mathbf{1} \\
\mathbf{2} \\
5 \\
6\end{array}$ & $\frac{1}{w^{3}\left(w^{2}\right)^{5 / 2}}$ & $\begin{array}{l}\mathbf{1} \\
\mathbf{2} \\
5 \\
6\end{array}$ \\
\hline$w^{-2}$ & $\frac{\left(w^{2}\right)^{5 / 2}}{w^{2}}$ & $\begin{array}{l}\mathbf{1} \\
\mathbf{2} \\
3 \\
4 \\
5 \\
6 \\
\end{array}$ & $\frac{\left(w^{2}\right)^{3 / 2}}{w^{2}}$ & $\begin{array}{l}\mathbf{1} \\
\mathbf{2} \\
3 \\
4 \\
5 \\
6\end{array}$ & $\frac{1}{\sqrt{\frac{1}{w^{2}}} w^{2}}$ & $\begin{array}{l}2 \\
3 \\
4 \\
6 \\
8\end{array}$ & $\frac{\sqrt{\frac{1}{w^{2}}}}{w^{2}}$ & $\begin{array}{l}\mathbf{2} \\
4 \\
6\end{array}$ & $\frac{1}{w^{2}\left(w^{2}\right)^{3 / 2}}$ & $\begin{array}{l}\mathbf{1} \\
\mathbf{2} \\
4 \\
5 \\
6\end{array}$ & $\frac{1}{w^{2}\left(w^{2}\right)^{5 / 2}}$ & $\begin{array}{l}1 \\
\mathbf{2} \\
4 \\
5 \\
6\end{array}$ \\
\hline$w^{-1}$ & $\frac{\left(w^{2}\right)^{5 / 2}}{w}$ & \begin{tabular}{|l|}
$\mathbf{1}$ \\
$\mathbf{2}$ \\
3 \\
5 \\
5 \\
6
\end{tabular} & $\frac{\left(w^{2}\right)^{3 / 2}}{w}$ & \begin{tabular}{|l|}
$\mathbf{1}$ \\
$\mathbf{2}$ \\
3 \\
5 \\
6 \\
\end{tabular} & $\frac{1}{\sqrt{\frac{1}{w^{2}}} w}$ & $\begin{array}{l}2 \\
6 \\
8\end{array}$ & $\frac{\sqrt{\frac{1}{w^{2}}}}{w}$ & 2 & $\frac{1}{w\left(w^{2}\right)^{3 / 2}}$ & $\begin{array}{l}\mathbf{1} \\
\mathbf{2} \\
5 \\
6\end{array}$ & $\frac{1}{w\left(w^{2}\right)^{5 / 2}}$ & $\begin{array}{l}\mathbf{1} \\
\mathbf{2} \\
5 \\
6\end{array}$ \\
\hline$w^{0}$ & $\left(w^{2}\right)^{5 / 2}$ & $\begin{array}{l}1 \\
2\end{array}$ & $\left(w^{2}\right)^{3 / 2}$ & $\begin{array}{l}1 \\
2\end{array}$ & $\frac{1}{\sqrt{\frac{1}{w^{2}}}}$ & 2 & $\sqrt{\frac{1}{w^{2}}}$ & 2 & $\frac{1}{\left(w^{2}\right)^{3 / 2}}$ & $\begin{array}{l}1 \\
2\end{array}$ & $\frac{1}{\left(w^{2}\right)^{5 / 2}}$ & $\begin{array}{l}1 \\
2\end{array}$ \\
\hline$w^{1}$ & $w\left(w^{2}\right)^{5 / 2}$ & $\begin{array}{l}\mathbf{1} \\
\mathbf{2} \\
5 \\
6\end{array}$ & $w\left(w^{2}\right)^{3 / 2}$ & $\begin{array}{l}\mathbf{1} \\
\mathbf{2} \\
5 \\
6\end{array}$ & $\frac{w}{\sqrt{\frac{1}{w^{2}}}}$ & 2 & $\sqrt{\frac{1}{w^{2}}} w$ & 2 & $\frac{w}{\left(w^{2}\right)^{3 / 2}}$ & $\begin{array}{l}\mathbf{1} \\
\mathbf{2} \\
3 \\
5 \\
6\end{array}$ & $\frac{w}{\left(w^{2}\right)^{5 / 2}}$ & $\begin{array}{l}\mathbf{1} \\
\mathbf{2} \\
3 \\
5 \\
6\end{array}$ \\
\hline$w^{2}$ & $w^{2}\left(w^{2}\right)^{5 / 2}$ & $\begin{array}{l}\mathbf{1} \\
\mathbf{2} \\
4 \\
5 \\
6\end{array}$ & $w^{2}\left(w^{2}\right)^{3 / 2}$ & $\begin{array}{l}\mathbf{1} \\
\mathbf{2} \\
4 \\
5 \\
6\end{array}$ & $\frac{w^{2}}{\sqrt{\frac{1}{w^{2}}}}$ & $\begin{array}{l}2 \\
4 \\
6\end{array}$ & $\sqrt{\frac{1}{w^{2}}} w^{2}$ & $\begin{array}{l}2 \\
4 \\
6\end{array}$ & $\frac{w^{2}}{\left(w^{2}\right)^{3 / 2}}$ & $\begin{array}{l}\mathbf{1} \\
\mathbf{2} \\
3 \\
4 \\
5\end{array}$ & $\frac{w^{2}}{\left(w^{2}\right)^{5 / 2}}$ & $\begin{array}{l}\mathbf{1} \\
\mathbf{2} \\
3 \\
4 \\
5 \\
6\end{array}$ \\
\hline$w^{3}$ & $w^{3}\left(w^{2}\right)^{5 / 2}$ & $\begin{array}{l}\mathbf{1} \\
\mathbf{2} \\
5 \\
6\end{array}$ & $w^{3}\left(w^{2}\right)^{3 / 2}$ & $\begin{array}{l}\mathbf{1} \\
\mathbf{2} \\
5 \\
6\end{array}$ & $\frac{w^{3}}{\sqrt{\frac{1}{w^{2}}}}$ & 2 & $\sqrt{\frac{1}{w^{2}}} w^{3}$ & & $\frac{w^{3}}{\left(w^{2}\right)^{3 / 2}}$ & $\begin{array}{l}\mathbf{1} \\
\mathbf{2} \\
3 \\
5\end{array}$ & $\frac{w^{3}}{\left(w^{2}\right)^{5 / 2}}$ & \begin{tabular}{l|l}
$\mathbf{1}$ & $\mathbf{2}$ \\
3 \\
5 \\
6
\end{tabular} \\
\hline
\end{tabular}


Table 14: Maxima 5.24 fullratsimp(...) for 1 st row $\times 1$ st column, with flaw numbers. Compare with Table 8 .

\begin{tabular}{|c|c|c|c|c|c|c|c|c|c|c|c|c|}
\hline $\overrightarrow{\downarrow \vec{x}}$ & \multicolumn{2}{|c|}{$\left(w^{-2}\right)^{-5 / 2}$} & \multicolumn{2}{|c|}{$\left(w^{-2}\right)^{-3 / 2}$} & \multicolumn{2}{|c|}{$\left(w^{-2}\right)^{-1 / 2}$} & \multicolumn{2}{|c|}{$\left(w^{-2}\right)^{1 / 2}$} & \multicolumn{2}{|c|}{$\left(w^{-2}\right)^{3 / 2}$} & \multicolumn{2}{|c|}{$\left(w^{-2}\right)^{5 / 2}$} \\
\hline$w^{-3}$ & $w \sqrt{w^{2}}$ & $\begin{array}{l}1 \\
6\end{array}$ & $\frac{\sqrt{w^{2}}}{w}$ & $\begin{array}{l}1 \\
2 \\
6\end{array}$ & $\frac{1}{\sqrt{\frac{1}{w^{2}}} w^{3}}$ & 2 & $\frac{\sqrt{\frac{1}{w^{2}}}}{w^{3}}$ & 2 & $\frac{1}{w^{5} \sqrt{w^{2}}}$ & $\begin{array}{l}1 \\
2 \\
6\end{array}$ & $\frac{1}{w^{7} \sqrt{w^{2}}}$ & $\begin{array}{l}\mathbf{1} \\
\mathbf{2} \\
6\end{array}$ \\
\hline$w^{-2}$ & $w^{2} \sqrt{w^{2}}$ & $\begin{array}{l}1 \\
4 \\
6\end{array}$ & $\sqrt{w^{2}}$ & $\begin{array}{l}1 \\
2 \\
6\end{array}$ & $\frac{1}{\sqrt{\frac{1}{w^{2}}} w^{2}}$ & 3 & $\frac{\sqrt{\frac{1}{w^{2}}}}{w^{2}}$ & 2 & $\frac{1}{w^{4} \sqrt{w^{2}}}$ & $\begin{array}{l}\mathbf{1} \\
\mathbf{2} \\
4 \\
6\end{array}$ & $\frac{1}{w^{6} \sqrt{w^{2}}}$ & $\begin{array}{l}\mathbf{1} \\
\mathbf{2} \\
4 \\
6\end{array}$ \\
\hline$w^{-1}$ & $w^{3} \sqrt{w^{2}}$ & $\begin{array}{l}1 \\
6\end{array}$ & $w \sqrt{w^{2}}$ & $\begin{array}{l}1 \\
2 \\
6 \\
\end{array}$ & $\frac{1}{\sqrt{\frac{1}{w^{2}} w}}$ & 2 & $\frac{\sqrt{\frac{1}{w^{2}}}}{w}$ & 2 & $\frac{1}{w^{3} \sqrt{w^{2}}}$ & $\begin{array}{l}1 \\
2 \\
6\end{array}$ & $\frac{1}{w^{5} \sqrt{w^{2}}}$ & $\begin{array}{l}\mathbf{1} \\
\mathbf{2} \\
6\end{array}$ \\
\hline$w^{0}$ & $w^{4} \sqrt{w^{2}}$ & $\begin{array}{l}1 \\
4\end{array}$ & $w^{2} \sqrt{w^{2}}$ & $\begin{array}{l}1 \\
2 \\
4 \\
6\end{array}$ & $\frac{1}{\sqrt{\frac{1}{w^{2}}}}$ & 2 & $\sqrt{\frac{1}{w^{2}}}$ & 2 & $\frac{1}{w^{2} \sqrt{w^{2}}}$ & $\begin{array}{l}1 \\
2 \\
4 \\
6\end{array}$ & $\frac{1}{w^{4} \sqrt{w^{2}}}$ & $\begin{array}{l}1 \\
2 \\
4 \\
6\end{array}$ \\
\hline$w^{1}$ & $w^{5} \sqrt{w^{2}}$ & $\begin{array}{l}1 \\
6\end{array}$ & $w^{3} \sqrt{w^{2}}$ & $\begin{array}{l}1 \\
2 \\
6\end{array}$ & $\frac{w}{\sqrt{\frac{1}{w^{2}}}}$ & 2 & $\sqrt{\frac{1}{w^{2}} w}$ & 2 & $\frac{1}{w \sqrt{w^{2}}}$ & $\begin{array}{l}\mathbf{1} \\
\mathbf{2} \\
6 \\
\end{array}$ & $\frac{1}{w^{3} \sqrt{w^{2}}}$ & $\begin{array}{l}\mathbf{1} \\
\mathbf{2} \\
6\end{array}$ \\
\hline$w^{2}$ & $w^{6} \sqrt{w^{2}}$ & $\begin{array}{l}1 \\
4 \\
6\end{array}$ & $w^{4} \sqrt{w^{2}}$ & $\begin{array}{l}1 \\
2 \\
4 \\
6 \\
\end{array}$ & $\frac{w^{2}}{\sqrt{\frac{1}{w^{2}}}}$ & $\begin{array}{l}2 \\
4 \\
6\end{array}$ & $\sqrt{\frac{1}{w^{2}}} w^{2}$ & 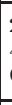 & $\frac{1}{\sqrt{w^{2}}}$ & $\begin{array}{l}\mathbf{1} \\
\mathbf{2} \\
4 \\
6 \\
\end{array}$ & $\frac{1}{w^{2} \sqrt{w^{2}}}$ & $\begin{array}{l}\mathbf{1} \\
\mathbf{2} \\
4 \\
6 \\
\end{array}$ \\
\hline$w^{3}$ & $w^{7} \sqrt{w^{2}}$ & $\begin{array}{l}1 \\
6\end{array}$ & $w^{5} \sqrt{w^{2}}$ & $\begin{array}{l}1 \\
\mathbf{2} \\
6\end{array}$ & $\frac{w^{3}}{\sqrt{\frac{1}{w^{2}}}}$ & 2 & $\sqrt{\frac{1}{w^{2}}} w^{3}$ & 3 & $\frac{w}{\sqrt{w^{2}}}$ & $\begin{array}{l}1 \\
2 \\
6\end{array}$ & $\frac{1}{w \sqrt{w^{2}}}$ & $\begin{array}{l}1 \\
2 \\
6\end{array}$ \\
\hline
\end{tabular}

Table 15: Unflawed results of Maple simplify(...) for 1st row $\times 1$ st column - a variant of form 4 .

Compare with Table 8 .

\begin{tabular}{|c||c|c|c|c|c|c|}
\hline$\vec{\triangleright}$ & $\left(w^{-2}\right)^{-5 / 2}$ & $\left(w^{-2}\right)^{-3 / 2}$ & $\left(w^{-2}\right)^{-1 / 2}$ & $\left(w^{-2}\right)^{1 / 2}$ & $\left(w^{-2}\right)^{3 / 2}$ & $\left(w^{-2}\right)^{5 / 2}$ \\
\hline \hline$w^{-3}$ & $w^{2} \operatorname{csgn}\left(\frac{1}{w}\right)$ & $\operatorname{csgn}\left(\frac{1}{w}\right)$ & $\frac{\operatorname{csgn}\left(\frac{1}{w}\right)}{w^{2}}$ & $\frac{\operatorname{csgn}\left(\frac{1}{w}\right)}{w^{4}}$ & $\frac{\operatorname{csgn}\left(\frac{1}{w}\right)}{w^{6}}$ & $\frac{\operatorname{csgn}\left(\frac{1}{w}\right)}{w^{8}}$ \\
\hline$w^{-2}$ & $w^{3} \operatorname{csgn}\left(\frac{1}{w}\right)$ & $w \operatorname{csgn}\left(\frac{1}{w}\right)$ & $\frac{\operatorname{csgn}\left(\frac{1}{w}\right)}{w}$ & $\frac{\operatorname{csgn}\left(\frac{1}{w}\right)}{w^{3}}$ & $\frac{\operatorname{csgn}\left(\frac{1}{w}\right)}{w^{5}}$ & $\frac{\operatorname{csgn}\left(\frac{1}{w}\right)}{w^{7}}$ \\
\hline$w^{-1}$ & $w^{4} \operatorname{csgn}\left(\frac{1}{w}\right)$ & $w^{2} \operatorname{csgn}\left(\frac{1}{w}\right)$ & $\operatorname{csgn}\left(\frac{1}{w}\right)$ & $\frac{\operatorname{csgn}\left(\frac{1}{w}\right)}{w^{2}}$ & $\frac{\operatorname{csgn}\left(\frac{1}{w}\right)}{w^{4}}$ & $\frac{\operatorname{csgn}\left(\frac{1}{w}\right)}{w^{6}}$ \\
\hline$w^{0}$ & $w^{5} \operatorname{csgn}\left(\frac{1}{w}\right)$ & $w^{3} \operatorname{csgn}\left(\frac{1}{w}\right)$ & $w \operatorname{csgn}\left(\frac{1}{w}\right)$ & $\frac{\operatorname{csgn}\left(\frac{1}{w}\right)}{w}$ & $\frac{\operatorname{csgn}\left(\frac{1}{w}\right)}{w^{3}}$ & $\frac{\operatorname{csgn}\left(\frac{1}{w}\right)}{w^{5}}$ \\
\hline$w^{1}$ & $w^{6} \operatorname{csgn}\left(\frac{1}{w}\right)$ & $w^{4} \operatorname{csgn}\left(\frac{1}{w}\right)$ & $w^{2} \operatorname{csgn}\left(\frac{1}{w}\right)$ & $\operatorname{csgn}\left(\frac{1}{w}\right)$ & $\frac{\operatorname{csgn}\left(\frac{1}{w}\right)}{w^{2}}$ & $\frac{\operatorname{csgn}\left(\frac{1}{w}\right)}{w^{4}}$ \\
\hline$w^{2}$ & $w^{7} \operatorname{csgn}\left(\frac{1}{w}\right)$ & $w^{5} \operatorname{csgn}\left(\frac{1}{w}\right)$ & $w^{3} \operatorname{csgn}\left(\frac{1}{w}\right)$ & $w \operatorname{csgn}\left(\frac{1}{w}\right)$ & $\frac{\operatorname{csgn}\left(\frac{1}{w}\right)}{w}$ & $\frac{\operatorname{csgn}\left(\frac{1}{w}\right)}{w^{3}}$ \\
\hline$w^{3}$ & $w^{8} \operatorname{csgn}\left(\frac{1}{w}\right)$ & $w^{6} \operatorname{csgn}\left(\frac{1}{w}\right)$ & $w^{4} \operatorname{csgn}\left(\frac{1}{w}\right)$ & $w^{2} \operatorname{csgn}\left(\frac{1}{w}\right)$ & $\operatorname{csgn}\left(\frac{1}{w}\right)$ & $\frac{\operatorname{csgn}\left(\frac{1}{w}\right)}{w^{2}}$ \\
\hline
\end{tabular}


Table 16: Simplification of $w / \sqrt{w^{2}}$, with flaw numbers

\begin{tabular}{|c|c||c|c|}
\hline system & transformations & \multicolumn{2}{|c|}{ input $\frac{w}{\sqrt{w^{2}}}$} \\
\hline \hline & Appendix rewrite rules & $\frac{\sqrt{w^{2}}}{w}$ & \\
\hline Mathematica & default & $\frac{w}{\sqrt{w^{2}}}$ & $\mathbf{2 , 6}, 8$ \\
\hline Mathematica & FullSimplify $(\ldots)$ & $\frac{w}{\sqrt{w^{2}}}$ & 6,8 \\
\hline Derive & default & $\frac{\sqrt{w^{2}}}{w}$ & \\
\hline TI-CAS & default & $\frac{w}{\sqrt{w^{2}}}$ & $\mathbf{2 , 6 , 8}$ \\
\hline Maple & default & $\frac{w}{\sqrt{w^{2}}}$ & $\mathbf{2 , 6 , 8}$ \\
\hline Maxima & default & $\frac{w}{\sqrt{w^{2}}}$ & $\mathbf{2 , 6 , 8}$ \\
\hline Maxima & fullratsimp $(\ldots)$ & $\frac{w}{\sqrt{w^{2}}}$ & 6,8 \\
\hline Maple & $\operatorname{simplify}(\ldots)$ & $\operatorname{csgn}(w)$ & \\
\hline
\end{tabular}

Table 17: Simplification of $\sqrt{w^{2}} / w-(-1)^{(1 / 2)\left(\arg \left(w^{2}\right)-2 \arg (w)\right) / \pi}$, with flaw numbers.

\begin{tabular}{|c|c|c|}
\hline system and transformation & result and flaw numbers & \\
\hline Appendix rewrite rules & $-i^{\left(-2 \operatorname{Arg}[w]+\operatorname{Arg}\left[w^{2}\right]\right) / \pi}+\frac{\sqrt{w^{2}}}{w}$ & 2 \\
\hline Mathematica default & $-i^{\left(-2 \operatorname{Arg}[w]+\operatorname{Arg}\left[w^{2}\right]\right) / \pi}+\frac{\sqrt{w^{2}}}{w}$ & 2 \\
\hline Mathematica FullSimplify(...) & $-i^{\left(-2 \operatorname{Arg}[w]+\operatorname{Arg}\left[w^{2}\right]\right) / \pi}+\frac{\sqrt{w^{2}}}{w}$ & 2 \\
\hline Derive default & $\frac{\sqrt{w^{2}}}{w}-\operatorname{IF}\left(w=0,0,(-1)^{\left(\operatorname{PHASE}\left(w^{2}\right)-2 \operatorname{PHASE}(w)\right) /(2 \pi)}\right)$ & 2 \\
\hline TI-CAS, default & $\begin{aligned} \pi i \begin{cases}0, & w=0 \\
\frac{\left(\operatorname{angle}\left(w^{2}\right)-2 \text { angle }(w)\right) 1 / 2}{w}-e^{2} & \text { else }\end{cases} \end{aligned}$ & 2 \\
\hline Maple default & $\frac{w}{\sqrt{w^{2}}}-(-1)^{\frac{1}{2} \frac{\operatorname{argument}\left(w^{2}\right)-2 \operatorname{argument}(z)}{\pi}}$ & 2 \\
\hline Maxima default & $\frac{w}{\sqrt{w^{2}}}-(-1)^{\frac{\operatorname{atan} 2(\sin (2 \operatorname{carg}(w)), \cos (2 \operatorname{carg}(w)))-2 \operatorname{carg}(w)}{2 \pi}}$ & 2 \\
\hline Maxima fullratsimp $(\ldots)$ & $\frac{\sqrt{w^{2}}(-1)^{\frac{\operatorname{carg}(w)}{\pi}}-w(-1)^{\frac{\operatorname{atan} 2(\sin (2 \operatorname{carg}(w)), \cos (2 \operatorname{carg}(w)))}{2 \pi}}}{w(-1)^{\frac{\operatorname{carg}(w)}{\pi}}}$ & 2 \\
\hline Maple simplify (...) & $\operatorname{csgn}(w)-(-1)^{\frac{1}{2} \frac{\operatorname{argument}\left(w^{2}\right)-2 \operatorname{argument}(z)}{\pi}}$ & 2 \\
\hline
\end{tabular}

